\title{
دقّة قياس الاختبارات التّكيفيّة متعدّة المراحل في ظل ظروف اختبارية مختلفة
}

\author{
ل لؤي شواشرة و محمود القرعان
}

Doi: //10.47015/17.2.8

\section{Assessing Measurement Accuracy of Multistage Adaptive Testing under Different Testing Modes}

Loiy Shawashreh and Mahmoud Alquraan, Yarmouk University, Jordan.

Abstract: This study aims at comparing the measurement accuracy of multistage adaptive testing under different conditions: test length (12, 24 and 36 items), panel design (13-3 and 1-2-2 panel designs), routing module length (long and short) and routing strategy (AMI and DPI). To achieve the aims of this study, (5000) true abilities were generated with a normal distribution of a mean of (1) and a standard deviation of (0). Measurement accuracy was assessed using mean of bias and mean squared error (MSE). The results show that the measurement accuracy increases as the test length increases, despite of panel design (1-3-3 and 1-2-2 panel designs), routing module length (long and short) or routing strategy. Moreover, the results show that panel design, routing strategy and routing module length marginally affect the measurement accuracy of multistage adaptive testing.

(Keywords: Multistage Adaptive Ttesting, Measurement Accuracy, Panel Design, Routing Module Length, Routing Strategy)

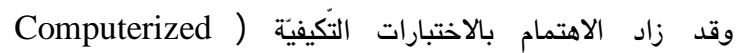
والاختبارات التكيفيّة متعدّة المراحل (Adaptive Testing: CAT مقابل اختبار الورقة (Multistage Adaptive Testing: MST) والقلم والاختبار الخطي المحوسب؛ نتيجةً للتطور التكنولوجي، واستخدام

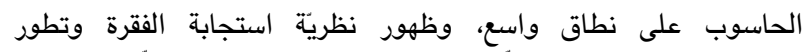

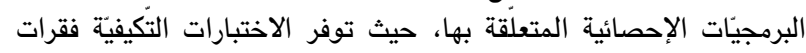

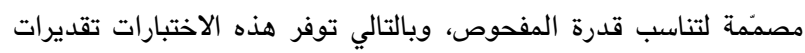

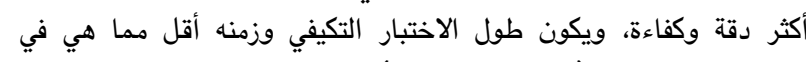

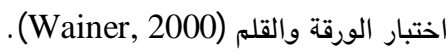

ملخص: هدفت الدرّاسة الحالية إلى مقارنة دقة قياس تصاميم مختلفة للاختبارات

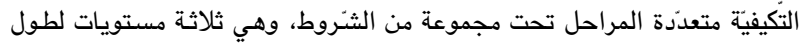

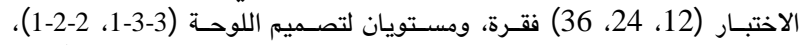

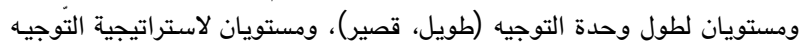
(AMI (DPI)

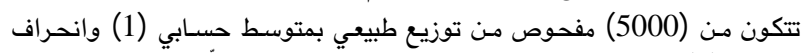

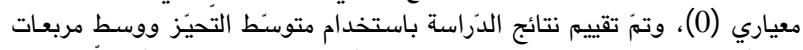

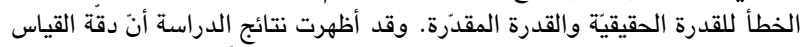

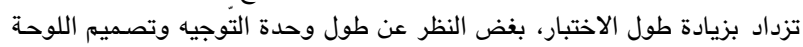

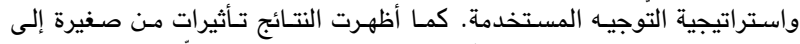

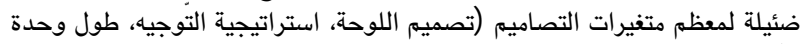
التوجيه)

(الكلمات المفتاحية: الاختبارات التكيفيَّة متعددة المراحل، دقّة القياس، تصميم اللوحة، استراتيجية التوجيه، طول وحدة الآتبار التيفية التوجيه)

مقدمة: يتزايد الاهتمام ببناء الاختبارات والمقاييس النفسية

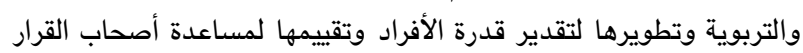

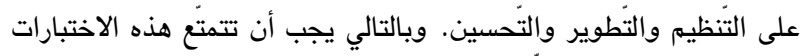

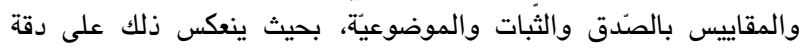

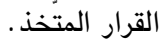

ولعقود طويلة، كانت اختبارات الورقة والقلم أكثر الطرق شيوعًا

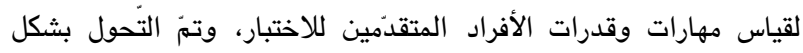

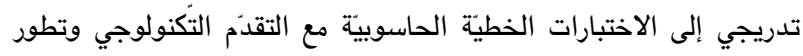

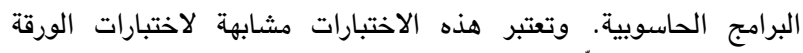

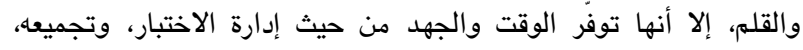

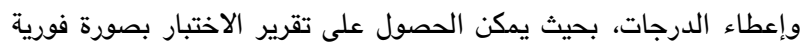
. (Yan, Von Davier \& Lewis, 2014)

ويتقدنم جميع المفحوصين في اختبارات الورقة والقلم والاختبار

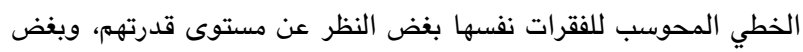

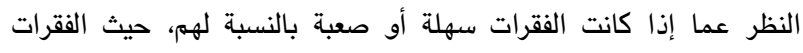

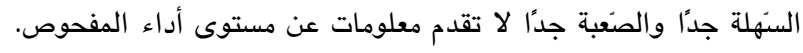

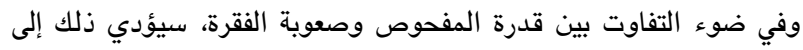

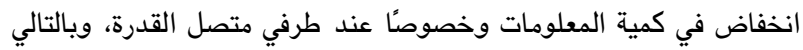
إلى انخفاض في دقة القياس (Zheng \& Chang, 2015) .

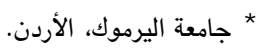
( حقوق الطبع محفوظة لجامعة اليرموك، إربد، الأردن. 
Magis et al., ) تستهدف مستويات قدرة محدّدة بدقة أكثر

ويوضح الثكل (1) مثالاً للوحة ذات تصميم 3-3-1، تتكون

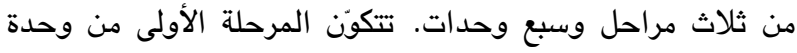

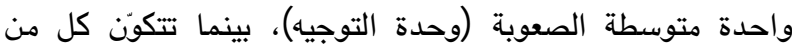

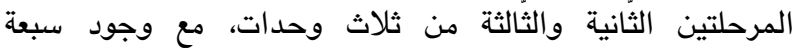
مسارات محتملة لكل مفحوص.

الشكل 1

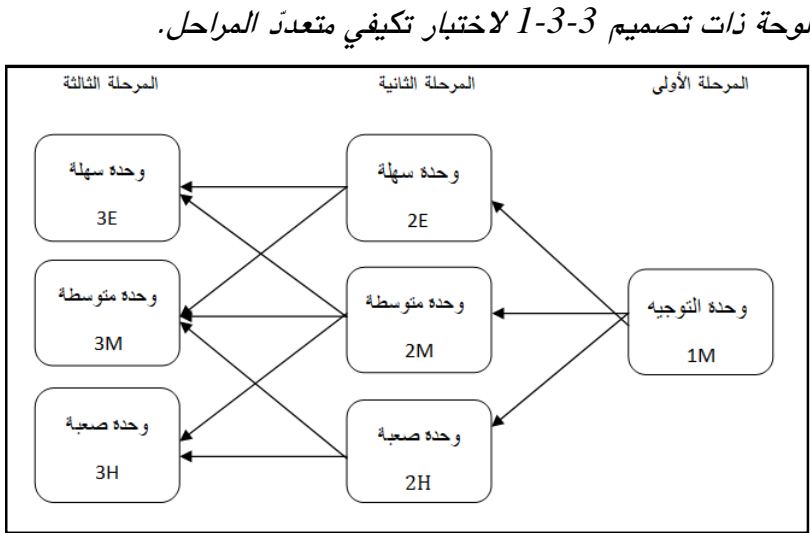

ويبدأ تنفيذ الاختبار باختيار لوحة عشوائيًا لكل مفحوص،

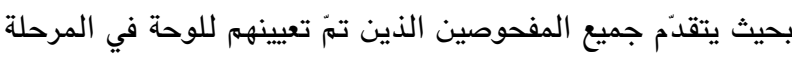

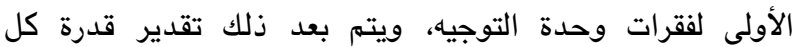

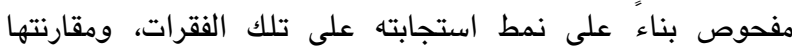
بمحكُّ محدّ مسبقًا لتوجيه المفحوصين إلى المرحلة الثانية. وتقدم للمفحوص في المرحلة الثانية وحدة جديدة من بين ثلاث وحدات المدات تتناسب مع مستوى قدرته المقدرة في المرحلة الأولى، ويتمّ تكرار الأمر في المرحلة الثالثة والأخيرة للوصول إلى التقدئ فلى التير النهائي للقدرة (Yan et al., 2014).

وتستخدم اختبارات MST التجميع الآلي للاختبار، مع التركيز

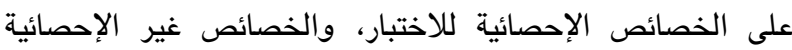

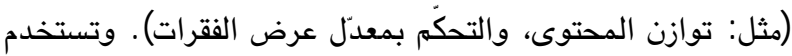

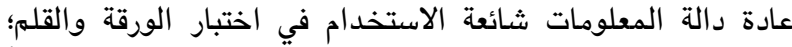
لأنها تسهل بناء صور متوازية للوحدات وتتيح استخدامًا فعالاً Armstrong \& Roussos, 2003; Melican ) لتجمع الفقرات .(et al., 2009

وثمة طرق تستخدم لبناء الألواح المتوازية في اختبارات

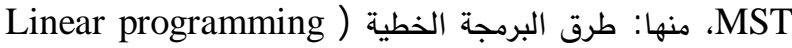

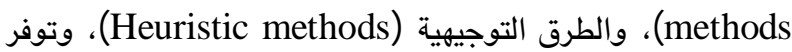

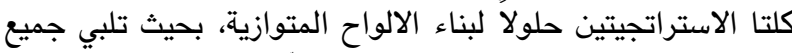

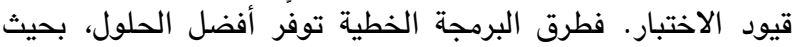
يمكنها تلبية جميع قيود الاختبار، بينما توفر الطرق التوفية التوجيهية التولية
كذلك بدأ الاهتمام بالاختبارات التكيفيّة متعددة المراحل في خمسينيات (Multistage Adaptive Testing: MST) القرن الماضي. وينظر إلى الاختبارات التكيفية متعددة المراحل على في التي

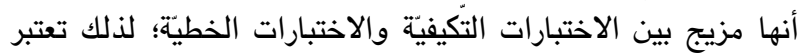

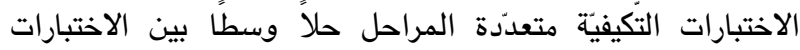

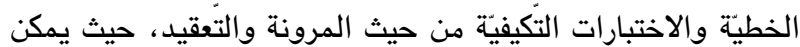

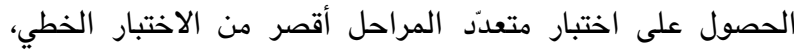

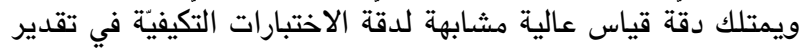
القدرة أو تصنيف المفحوصين (Zheng et al., 2012).

وتقدم لكل مفحوص في الاختبار التكيفي المحوسب فقرات

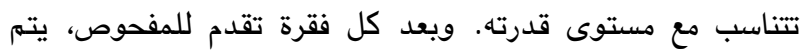
تقدير قدرته، وتقدم له فقرة جديدة تتناسب مع القدرة المقدرة فئرة

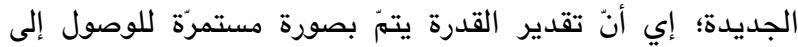

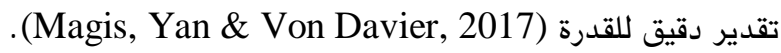
يتشابه تصميم MST مع تصميم CAT بحيث تكون الفقرات مناسبة لمستوى قدرة المفحوص، ويتكون اختبار MST من

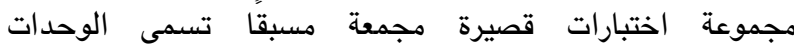
(Modules) ويتم توجيه المفحوصين إلى الوحدات اللاحقة بناء على قدرات التهمه المقدرة في الخطوة السابقة، وتغطي هذه الوحدات الوحدات مستويات مختلفة من الصعوبة (Yan et al., 2014).

وتتشابه المكونات الأساسية للاختبارات التكيفيّة متعددّة

المراحل والاختبارات التكيفيّة من حيث تجمع الفقرات، واستراتيجية

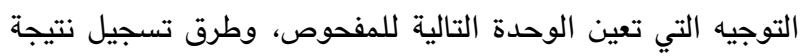
المفحوص، بينما تمتلك MST عوامل فريدة، مثل الوحدات والألواح والمراحل. تمثل الوحدة مجموعة من الفقرات يتم إنشاؤها

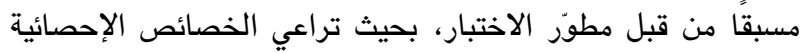

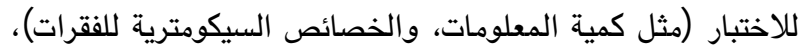

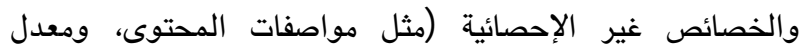
التعرض). ويتم دمج الوحدات لإنشاء اللوحة (Panel) التئ التئ تعتبر

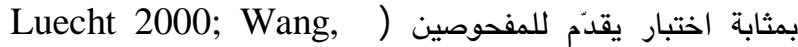

. (2017

ويبدأ تنفيذ اختبارMST بتقديم مجموعة من الفقرات تسمى وحدة التوجيه (Routing module) لجميع المفحوصين، ويتم بعد ذلك تقدير قدرة كل مفحوص بناء على نمط استجابته على هذه

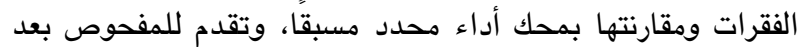

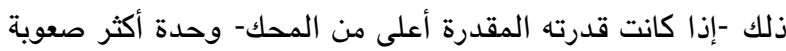

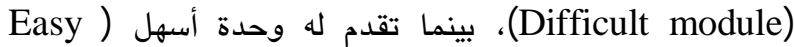

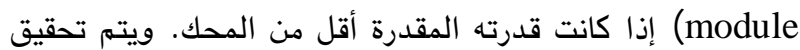

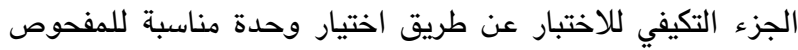

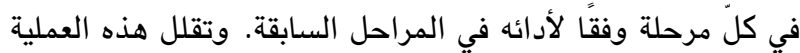

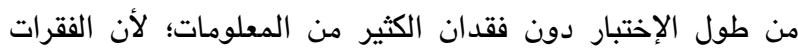


(al., 2015 التي أظهرت تفوق طريقة EAP على طريقة الأرجحية

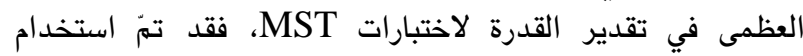
طريقة EAP في الدراسة الحالية. وأجرى جودون (Jodoin, 2003) دراسة هدفت إلى مقارنة

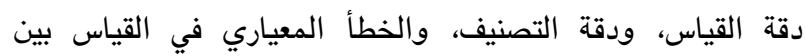

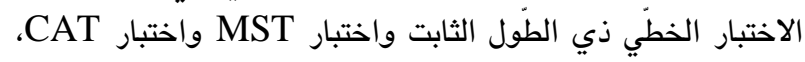

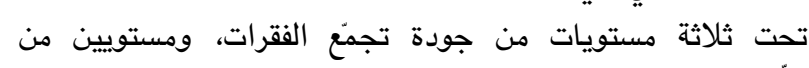

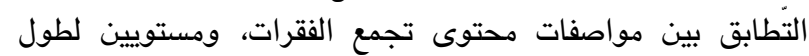

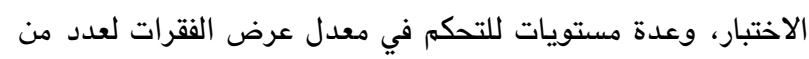

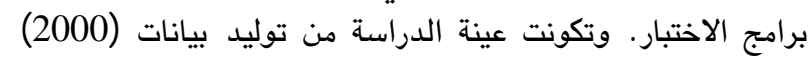

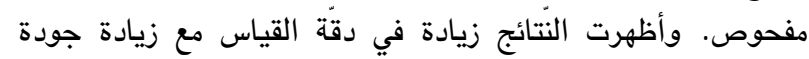
تجمع الفقرات و زيادة طول الاختبار .

وأجرى جودون وآخرون (Jodoin et al., 2006) دراسة

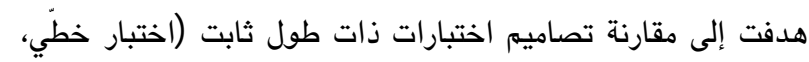

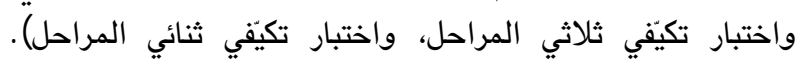

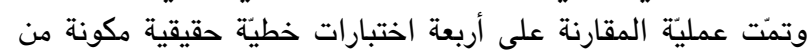

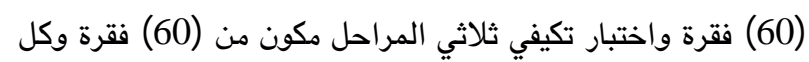

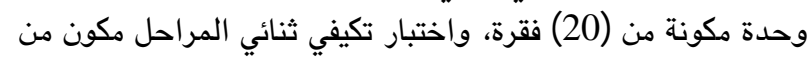

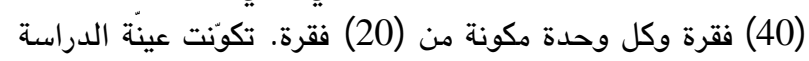

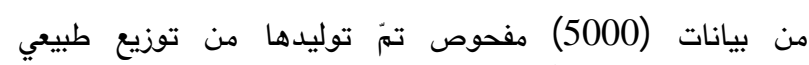

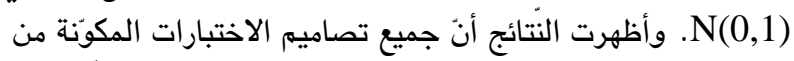

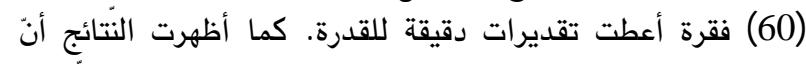

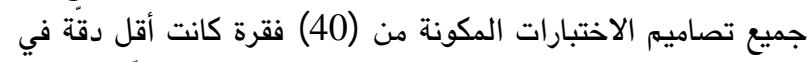

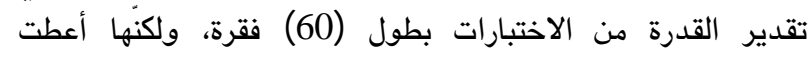
تقديرات ضمن النطاق المعقول والمقبول.

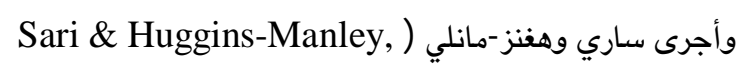
2017) دراسة هدفت إلى استكشاف دقة قياس

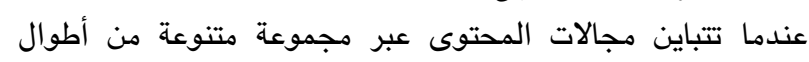

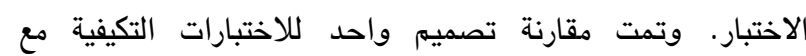

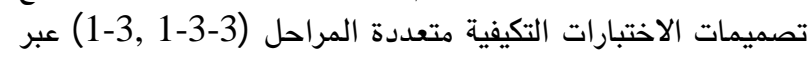

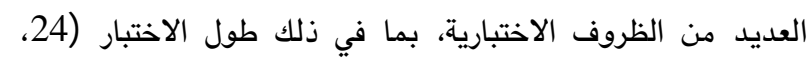

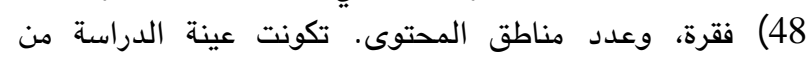

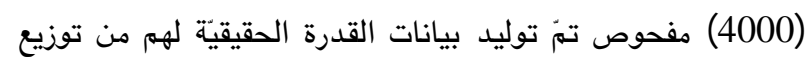
طييعي N(0,1). وأظهرت نتائج الدزراسة أنَ لطول الاختبار تأثيرًا في النتائج أكثر من عدد مناطق المحتوى.

وقام كيم وآخرون (Kim et al., 2013) بإجراء دراسة

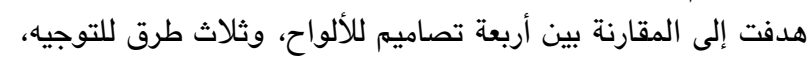

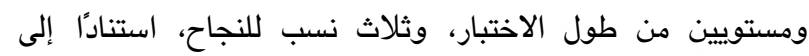

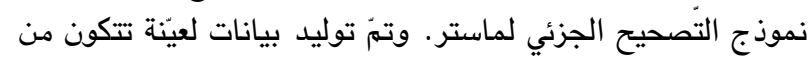

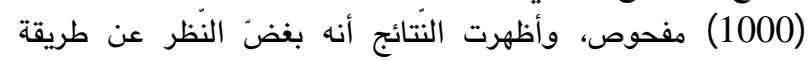

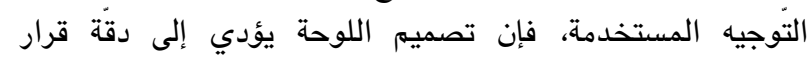

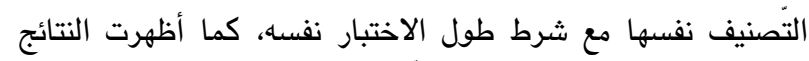
أنه بزيادة طول الاختبار، تزداد دقة القياس.
حلولاً قريبة من الحل الأمثل بتكلفة أقل، وإضافة إلى العديد من المزايا العمليّة ومنها سهولة الاستخدام (Zheng et al., 2012).

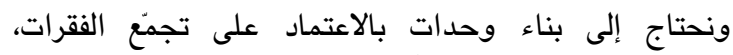

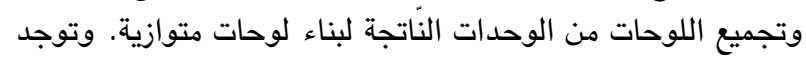

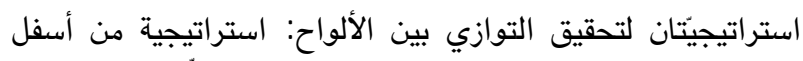

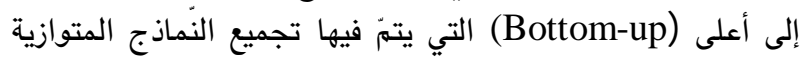

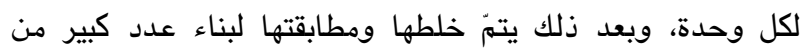
الألواح المتوازية، واستراتيجية من أعلى إلى أسفل (Top- down)

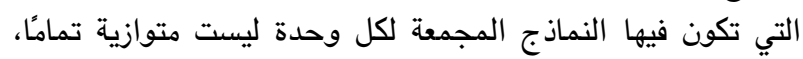

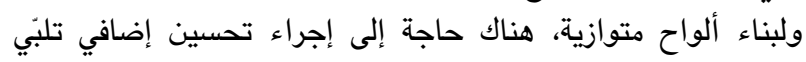

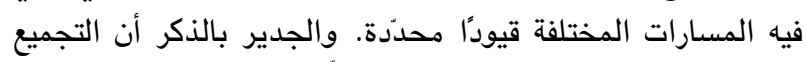

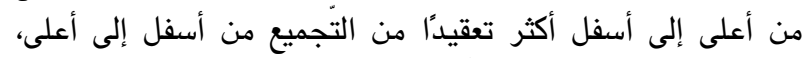

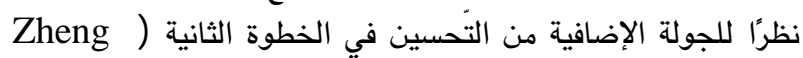
.(et al., 2012; Luecht \& Nungester, 1998

وتستخدم العديد من الأساليب للتجميع الآلي للاختبار منها:

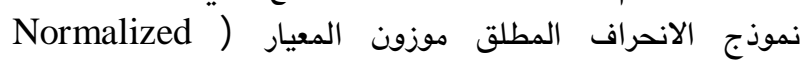
Weighted Absolute Deviation: NWAD Luecht, 2000) نموذج الانحراف الموزون ( Weighted Deviation ،(Swanson \& Stocking, 1993) .(Model: WDM)

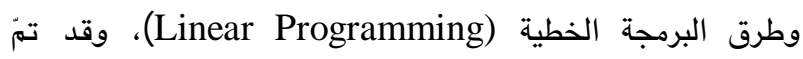
استخدام طرق البرمجة الخطيّة لتجميع الوحدات في هذه الدرّاسة (Diao \& van der Linden, 2011)

وتستخدم العديد من استراتيجيات التوجيه لتحديد نقطة التواليه

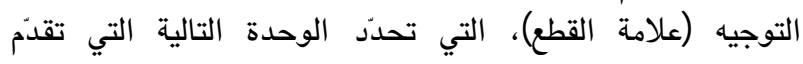

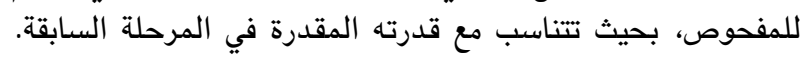

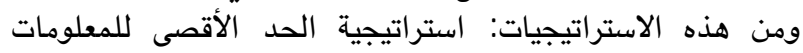
تصمل (Approximate Maximum Information: AMI) تعمل على تحديد نقط التقاطع بين دالَة معلومات الوحدة التي تمّ التمات

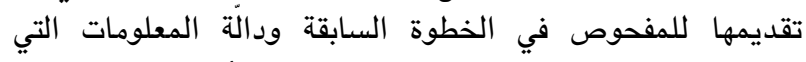

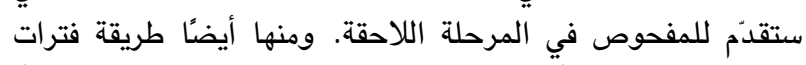
المجتمع المحدَة (Defined Population Interval: DPI)

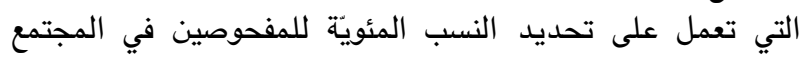

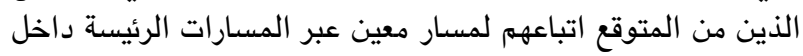
اللوحة (Luecht et al., 2006).

وتعتبر طرق تقدير القدرة خطوة أساسية في إجراءات

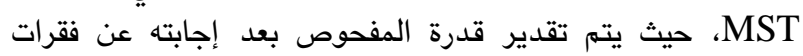

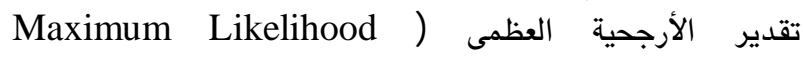
(Estimation: MLE

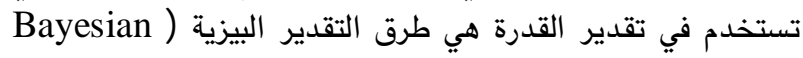
(Estimation التي تعتمد على معلومات التون التونع (Expected A Posterior: EAP)

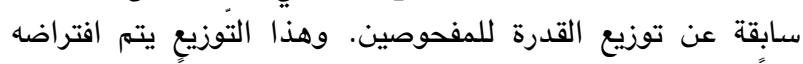

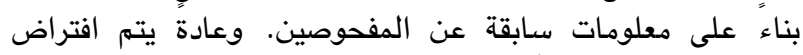

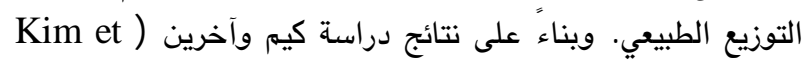


تقدير القدرة، كما أنّ زيادة عدد الوحدات من ثلاث إلى خمس أدت

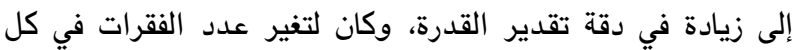
مرحلة تأثير ضئيل على دقة تقدير القدرة.

كما أجرى كيم وآخرون (Kim et al., 2015) دراسة هدفت

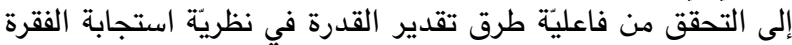

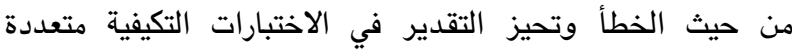

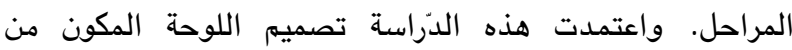
مرحلتين (3-1)، ومستويين لصعوبة الفقرات في المرحلة الثانية

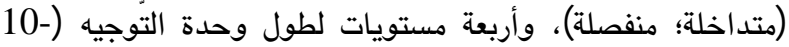
ما (30, 15-25, 20-20, 25-15)، وسبع طرق لتقدير القدرة؛ لمعرفة ما إذا كان هناك تفاعل بين طرق تقدير القدرة وتصميمات MST. تكونت عينّة الدراسة من بيانات القدرة الحقيقية لـ (8200)

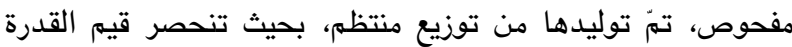
بين (-3) و (3) . وأظهرت نتائج الدرّاسة أنَ هناك تأثيرًا ضئيلاً

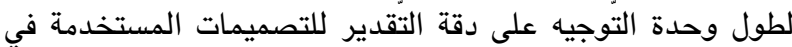

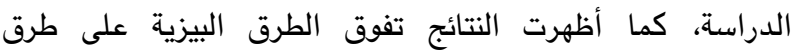

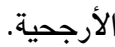

وأجرى أوزتورك (Oztürk, 2019) دراسة هدفت إلى فحص تأثير طول وحدة التوجيه وخصائصها المختلفة على دقة القياس.

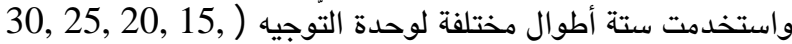
(10, 5 فقرة مع طول متغير للاختبار (50, 45, 40, 35,30, 25) فقرة، وتسع خصائص سيكومترية لفقرات وحدة التوجيه، وتصميمان مختلفان للوحة. وتكونت عينة الدراسة من محاكاة القدرة الحقيقية لـ لـ

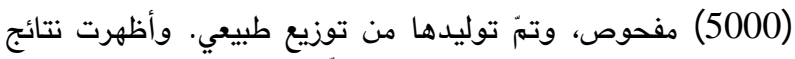

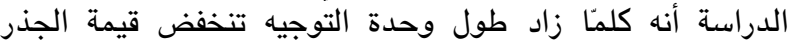

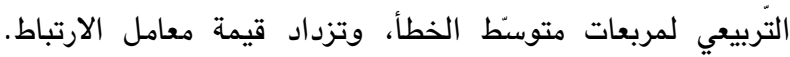

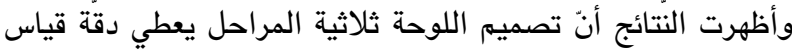

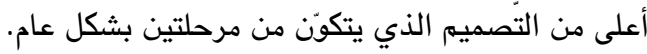

وبعد مراجعة الدراسات السابقة، تتضح قلة الدراسات التي

تناولت تصاميم الاختبارات متعدذة المراحل باستخدام اختبارات قصيرة، وأن أغلب الدراسات تناولتها باستخدام الاختبارات الطويلة

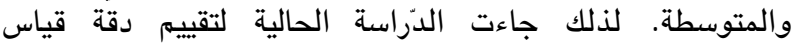
الاختبارات التكيفية متعددة المراحل باستخدام اختبارات قصيرة

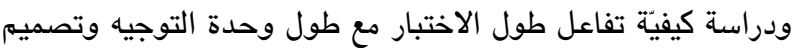

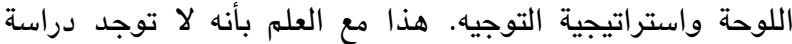

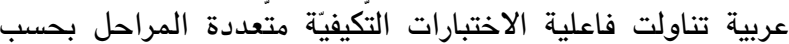

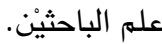

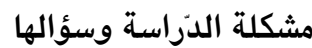

يهدف التقييم واسع النطاق (Large-scale Assessment) في التعليم أو المجالات الأخرى إلى توفير معلومات أساسية، مثل الناف

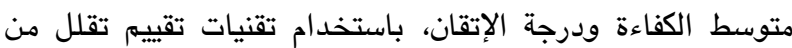

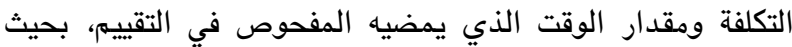

وأجرت وانغ (Wang, 2017) دراسة هدفت إلى المقارنة العادلة بين أداء الاختبارات التكيفيّة والاختبارات التكيفيّة متعددة

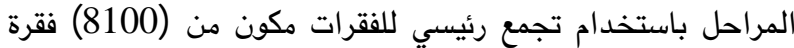

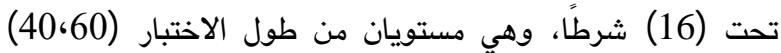

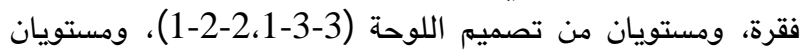
من استراتيجية التوجيه (AMI, DPI)، ومستويان من أولويية

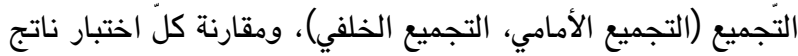

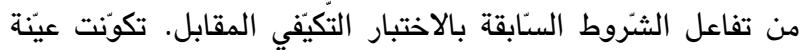
الدرّاسة من (5000) مفحوص، تمر توليد بيانات القدرة الحقيقيَّة

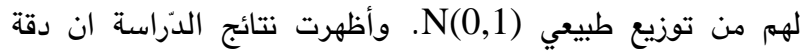
قياس MST تزداد بزيادة طول الاختبار. كما أظهرت النتائج تأثيرات ضئيلة لتصميم اللوحة وطريقة التوجيه، وأوصت بمقاريات بارنة دقة قياس تصاميم MST باستخدام اختبارات قصيرة. وأجرى زنزكي (Zenisky, 2004) دراسة هدفت إلى البحث في الآثار المترتبة على تفاعل العديد من متفيرات تصميم MST. وتمت دراسة المتفيرات الآتية: تصميم اللوحة وله العاديل أربعة مستويات

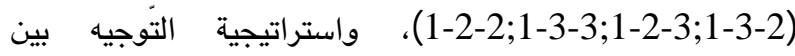
المراحل ولها أربعة مستويات، والنسب المئوييّة للتوجيه ولها ثلاثة مستويات. وتكونت عينة الدراسة من بيانات (9000) مفحوص تمز توليدها من توزيع طبيعي N(0,1). وأظهرت نتائج الدراسة تأثيرات من صغيرة إلى ضئيلة لمعظم متفيَرات التصاميم (تصميم اللوحة،

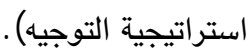

وأجرى زنزكي وهامبلتون ) Zenisky \& Hambleton, 2004) دراسة هدفت إلى استكثاف طريقة تفاعل متفيّرات تصاميم الاختبارات التكيفيّة متعددة المراحل مع بعضها البعض، وكيفيّة

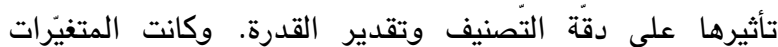

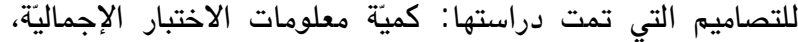

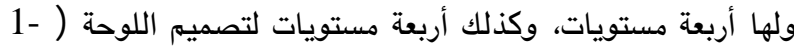

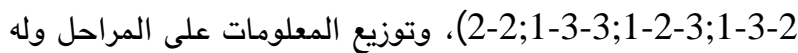

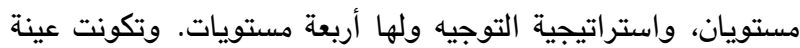

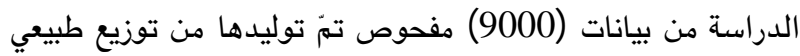
N(0,1) وأظهرت نتائج الدراسة تأثيرات من صغيرة إلى ضئيلة لمعظم متغيرات التصاميم (تصميم اللوحة، استراتيجية التوجيه).

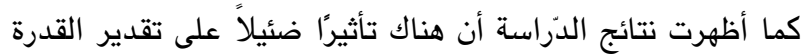
عندما تزيد كمية المعلومات على (10) بشكل كبير .

وقد سعت دراسة باتسولا (Patsula, 1999) إلى مقارنة دقة تقدير القدرة بين الاختبارات التكيفية والاختبارات التكيفية متعددة

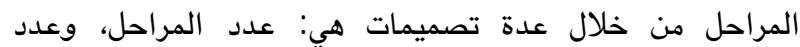

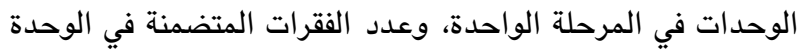

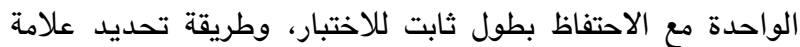

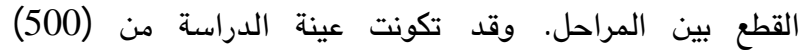

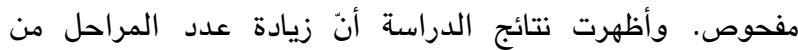
مرحلتين إلى ثلاث مراحل بشكل عام تقلل من حجم الخطأ في 


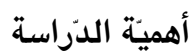

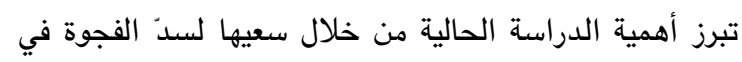

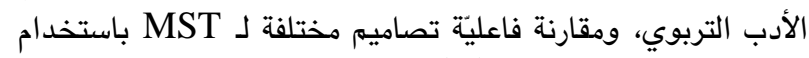

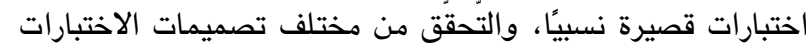

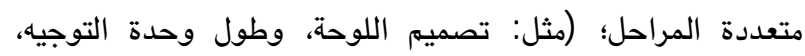

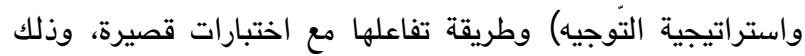
على العكس من الدراسات السابقة التي سعت إلى عملية المقارئ التعارنة باستخدام اختبارات طويلة .

$$
\text { محدّدات الدراسة }
$$

اقتصرت الدراسة الحالية على طرق تجميع محدَّة للاختبارات

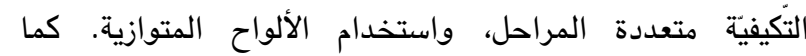

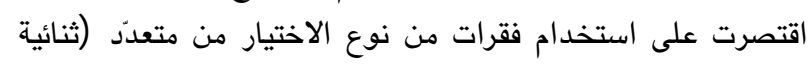

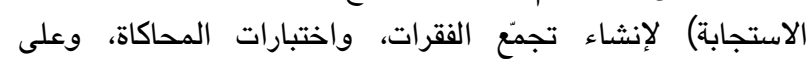
استخدام بيانات مولدة لبناء تجمع الفقرات، وبيانات القدات القدرة الحقيقية لعينة الدراسة.

الطريقة

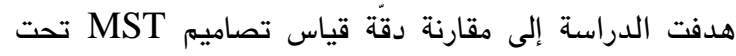

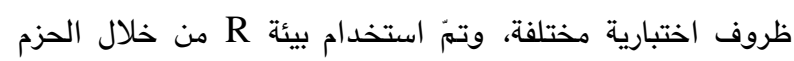
lpsolve MST

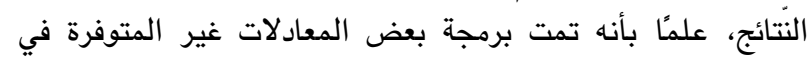

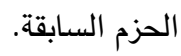

\section{MST توليد بيانات}

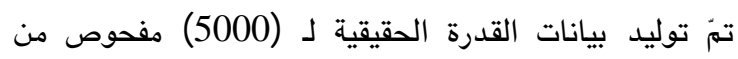

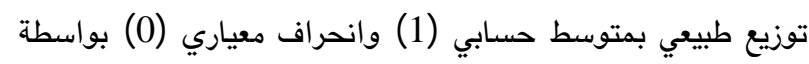

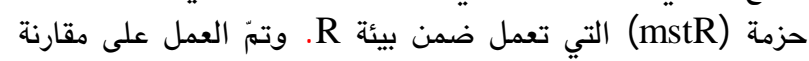

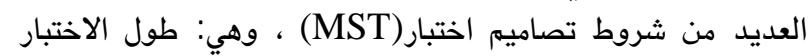

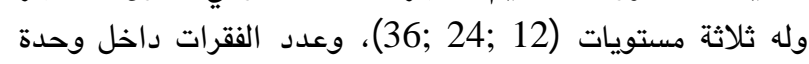
التوجيه وله مستويان (طويلة؛ قصيرة)، وتصميم اللوحة وله وله

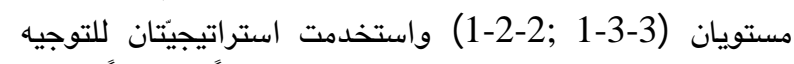
(AMI; DPI) عبر خمس لوحات متوازية . (MST)

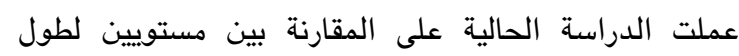

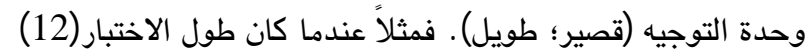

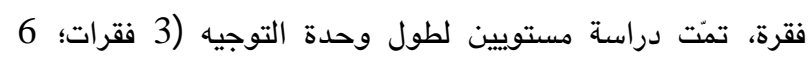

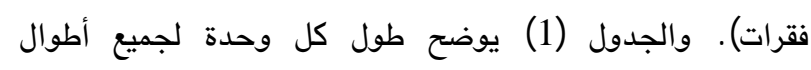

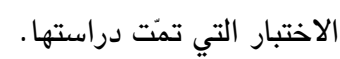

تساعد هذه المعلومات أصحاب القرار على تحسين النظم

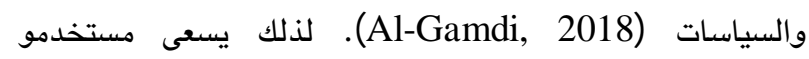

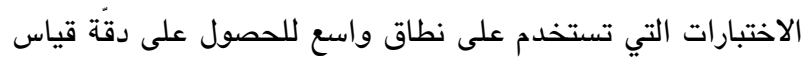

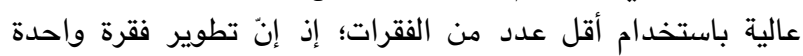

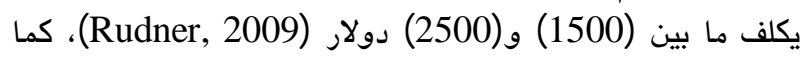
أن إدارة الاختبارات الطويلة وتنفيذها بحاجة إلى أوقات طويلة. ويلجأ مطورّو الاختبارات في بعض الأحيان إلى استخدام

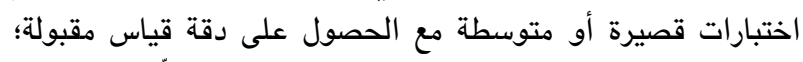

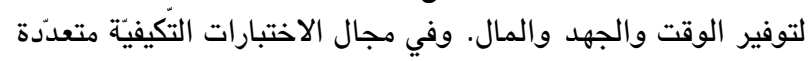

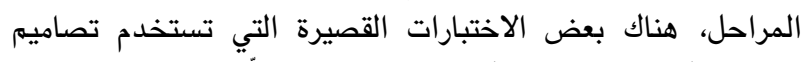
(Wang, 2017) MST التعليمي ( Assessment of Educational .(Progress: NAEP

ونتيجة لشيوع الاختبارات التكيفيّة متعدّدة المراحل

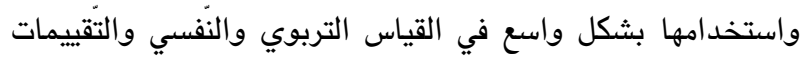

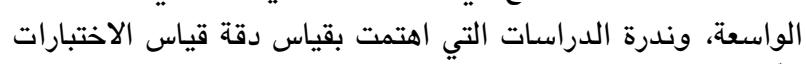

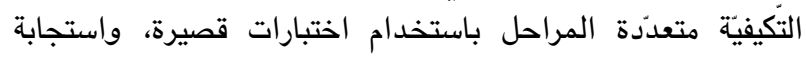

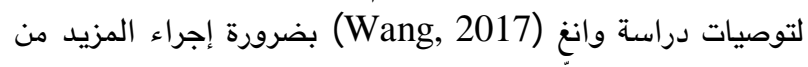

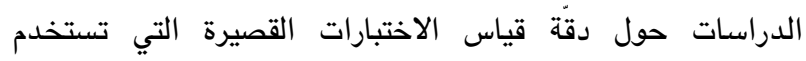

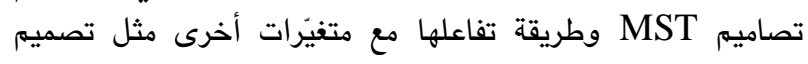
اللوحة وطول وحدة التوجيه. فإنّ الدراسة الحالية سعت للمقات المقارنة

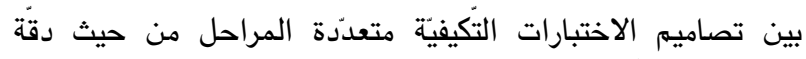

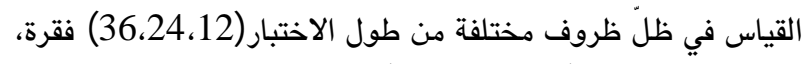

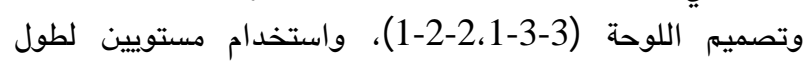

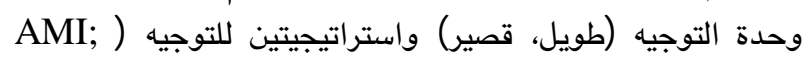

(DPI

وبالتحديد، فإنّ هذه الدزاسة تحاول الإجابة عن السؤال الآتي: هل تختلف دقة قياس تقدير القدرة للاختبارات التكّيفيّة

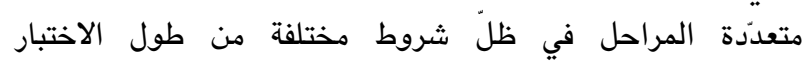

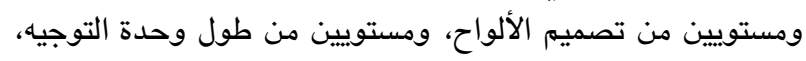
وإستراتيجيتين للتوجيه (AMI) و (DPI)

$$
\text { هدف الدراسة - مدراس }
$$

هدفت الدرّاسة الحالية إلى مقارنة دقة قياس تصاميم مختلفة

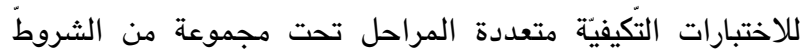

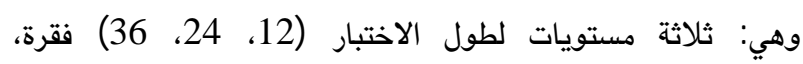

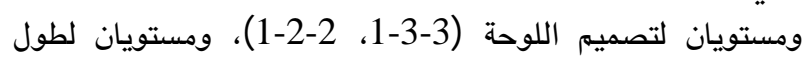

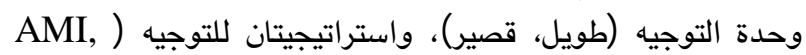
.(DPI 


\section{الجدول 2}

الجدول 1

توزيع معلمة الصعوية لجميع الوحدات.

\begin{tabular}{|c|c|c|}
\hline $1-2-2$ & $1-3-3$ & التصميم \\
\hline $\mathrm{N}(0,0.3)$ & $\mathrm{N}(0,1)$ & المرحلة الأولى \\
\hline $\mathrm{N}(-0.7,0.6)$ & $\mathrm{N}(-1,0.6)$ & المرحلة الثانية (2E) \\
\hline ---- & $\mathrm{N}(0,0.6)$ & المرحلة الثانية (2M) \\
\hline $\mathrm{N}(0.7,0.6)$ & $\mathrm{N}(1,0.6)$ & المرحلة الثانية (2H) \\
\hline $\mathrm{N}(-0.7,0.3)$ & $\mathrm{N}(-1,0.3)$ & المرحلة الثالثة (3E) \\
\hline ---- & $\mathrm{N}(0,0.3)$ & المرحلة الثالثة (3M) \\
\hline $\mathrm{N}(0.7,0.3)$ & $\mathrm{N}(1,0.3)$ & المرحلة الثالثة (3H) \\
\hline
\end{tabular}

وتمّ توليد معلمة الصنعوبة لوحدة التوجيه من توزيع طبيعي

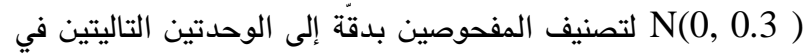
المرحلة الثانية لتصميم 2-2-1، ونظرًا لأنّ توزيع القدرة يتبع التونين التوزيع الطبيعي، فإن من المتوقع توجيه المفحوصين بعدد متساوٍ

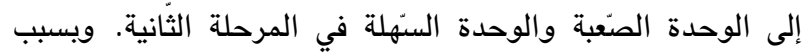

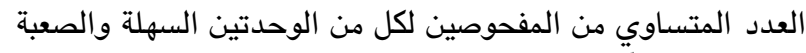

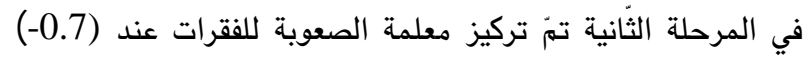

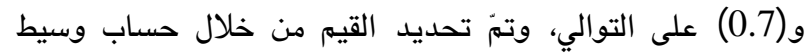
مستوى القدرة لنصفين من المفحوصين، وتم تحديد متوسيّ مئم

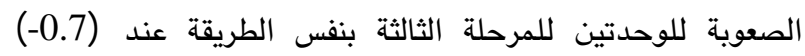

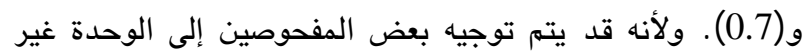

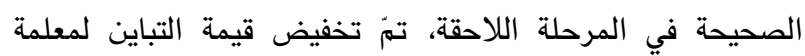
الصعوبة في المرحلة الثالثة ليكون هناك تداخل أعلى لتوزيع تلفي الصعوبة على مقياس القدرة (Wang, 2017) .

وقد تم توليد معلمة الصعوبة لوحدة التوجيه للتصميم -3-10

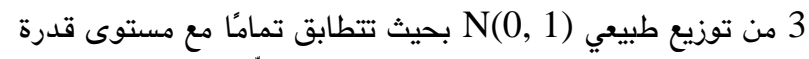

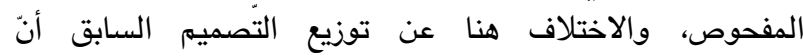
المفحوصين تم توجيههم إلى ثلاث وحدات في المرحلة الثانية. وتم توجيه المفحوصين في المرحلة الثانية والمرحلة الثالثة إلى ثلاث وحدات مختلفة بحيث تم تحديد متوسط صعوبة (1-)، (0)، (1)

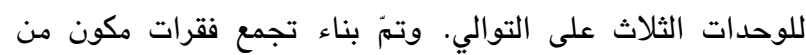

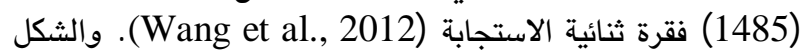

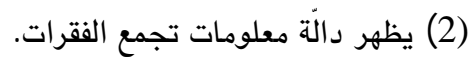

طول الوحدة لتصاميم MST

\begin{tabular}{|c|c|c|c|}
\hline \multicolumn{2}{|c|}{ تصميم اللوحة 3-3-1 } & \multicolumn{2}{|c|}{ تصميم اللوحة 2-2-1 } \\
\hline & عدد الفقرات & طا & عدد الفقرات \\
\hline لوقل الطبر & في الوحدة & لصقل التبر & في الوحدة \\
\hline 36 & $18-9-9$ & 36 & $18-9-9$ \\
\hline 36 & $9-9-18$ & 36 & $9-9-18$ \\
\hline 24 & $12-6-6$ & 24 & $12-6-6$ \\
\hline 24 & $6-6-12$ & 24 & $6-6-12$ \\
\hline 12 & $6-3-3$ & 12 & $6-3-3$ \\
\hline 12 & $3-3-6$ & 12 & $3-3-6$ \\
\hline
\end{tabular}

وتعتمد عمليّة تجميع الوحدات في MST على خصائص الفقرات المتوفرة في تجمع الفقرات. وللحصول على تجمّع فقرات

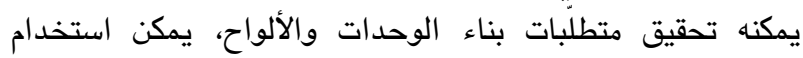
مجموعة من الاختبارات الثابتة المثلى التي تتوافق مع مستوى قدرة المفحوصين لبناء هذا التجمع ( Van der Linden \& (Veldkamp, 2006

تمّ بناء تجمع الفقرات باستخدام مجموعة من الاختبارات الثابتة المثلى التي تتوافق مع مستوى قدرة المفحوصين. وتم توليد تجمع الفقرات باستخدام النموذج ثلاثي المعلمة بحيث تكون الفقرات موني

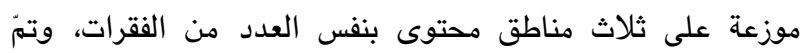

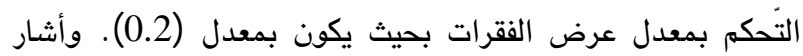

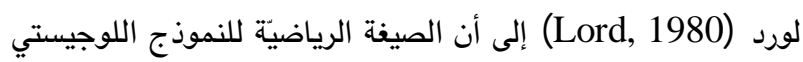
ثلاثي المعلمة تعطى بالعلاقة الآتية:

$$
P_{\mathrm{j}}(\theta)=c_{\mathrm{j}}+\left(1+\mathrm{c}_{\mathrm{j}}\right) \frac{1}{1+\mathrm{e}^{-\mathrm{Da}_{\mathrm{j}}\left(\theta-b_{j}\right)}}
$$

تمثل b مستوى صعوبة الفقرة، وa معلمة التمييز للفقرة، معثة

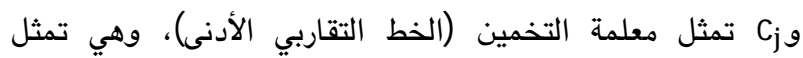
احتمال إجابة المفحوص إجابة صحيحة في حال غياب القدرة،

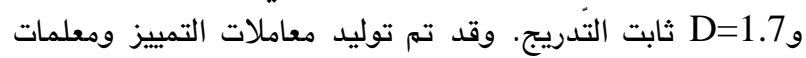
التخمين من التوزيعات c a lognormal (0, 0.3) على التوالي لمحاكاة تجمع فقرات اختبار Uniform (0.1, 0.2)

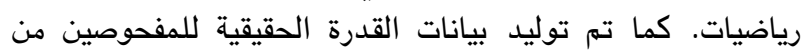
توزيع طبيعي) N(0, 1)، بينما تم توليد معلمة الصعوبة لتجمع توليد الفقرات من توزيع طبيعي للحصول على أفضل تطابق بين مستوى تولئ قدرة المفحوصين ومعلمة الصعوبة (Leung et al., 2005). والجدول (2) يوضح توزيع معلمة الصعوبة لكلّ وحدة. 
دالة معلومات تجمع الفقرات.

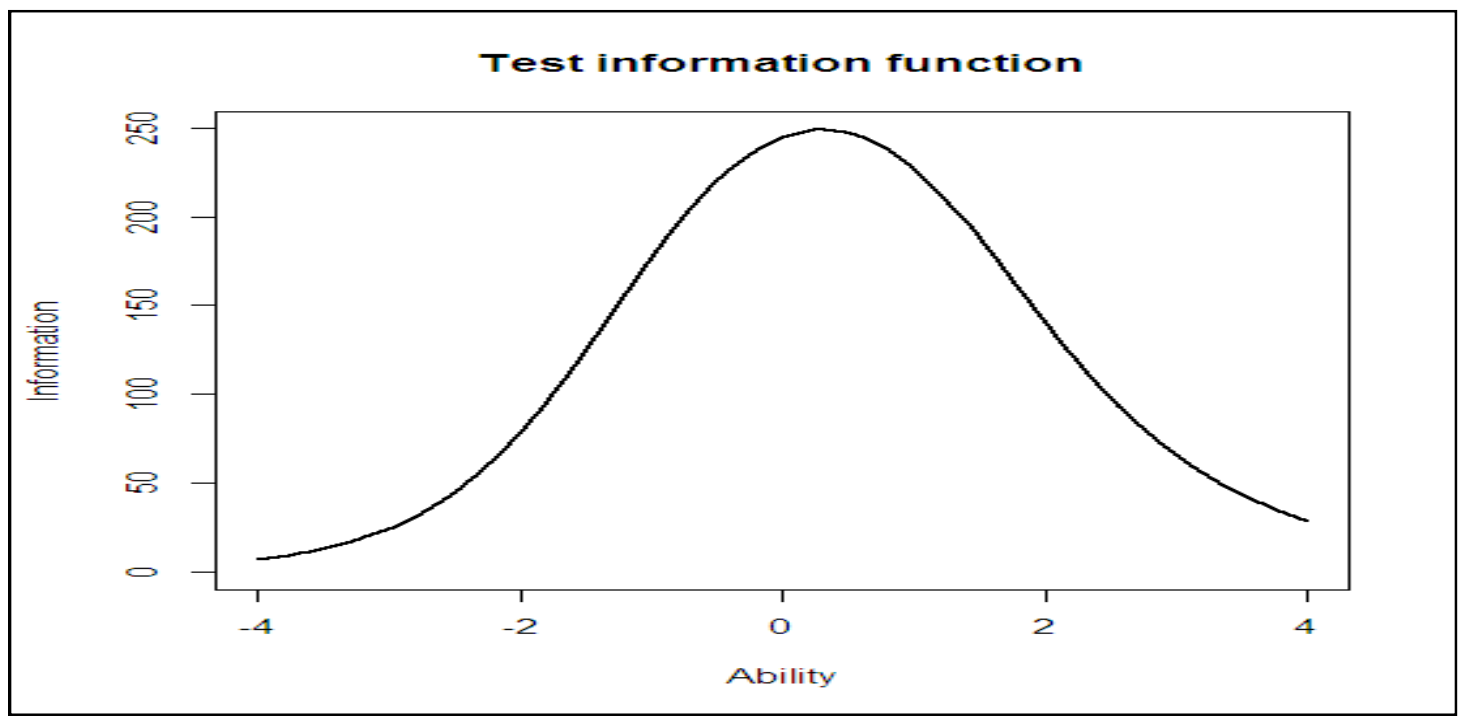

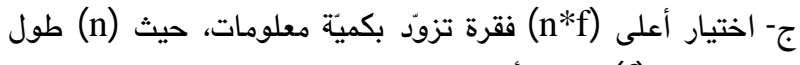
الوحدة، و(f) عدد الألواح المراد تكوينها.

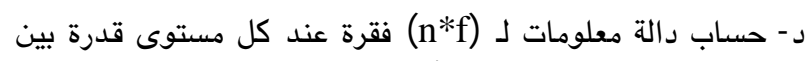

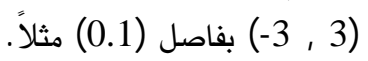

ودالة معلومات الهدف تعرف كما يأتي:

$$
\operatorname{TIF}\left(\theta_{j}\right)=\frac{\sum_{i=1}^{n * m} I_{I}\left(\theta_{j}\right)}{m}
$$

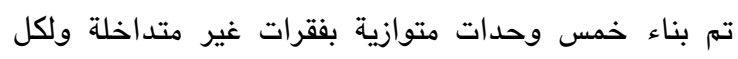

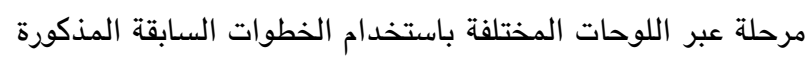

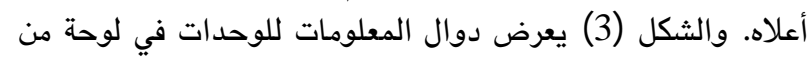
اللوحات ذات التصميم 3-3-1 الثكل (3) بعرض

تمزّ استخدام استراتيجية من أسفل إلى أعلى (Bottom-up)

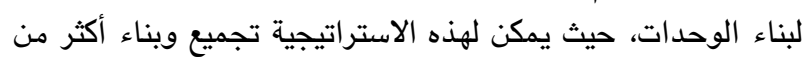

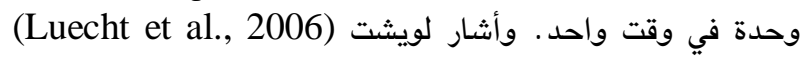

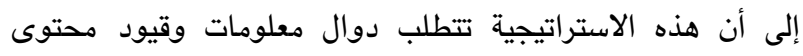

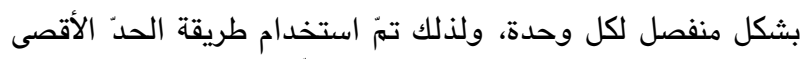

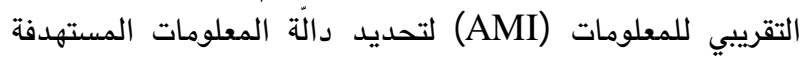

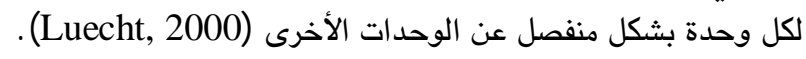

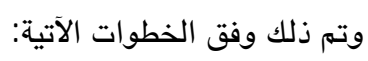

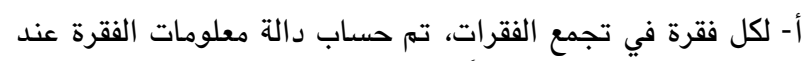

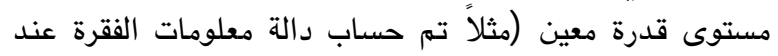
مستوى قدرة مقداره صفر لوحدة التوجيه).

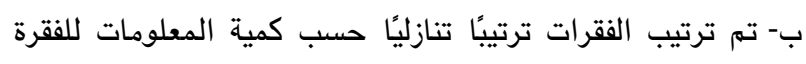

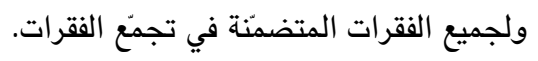

دالة المعلومات للوحدات لواحدة من اللوحات ذات التصميم 3-3-13؛ طول الاختبار (36) فقرة؛ طول وحدة التوجيه (18) نقرة .

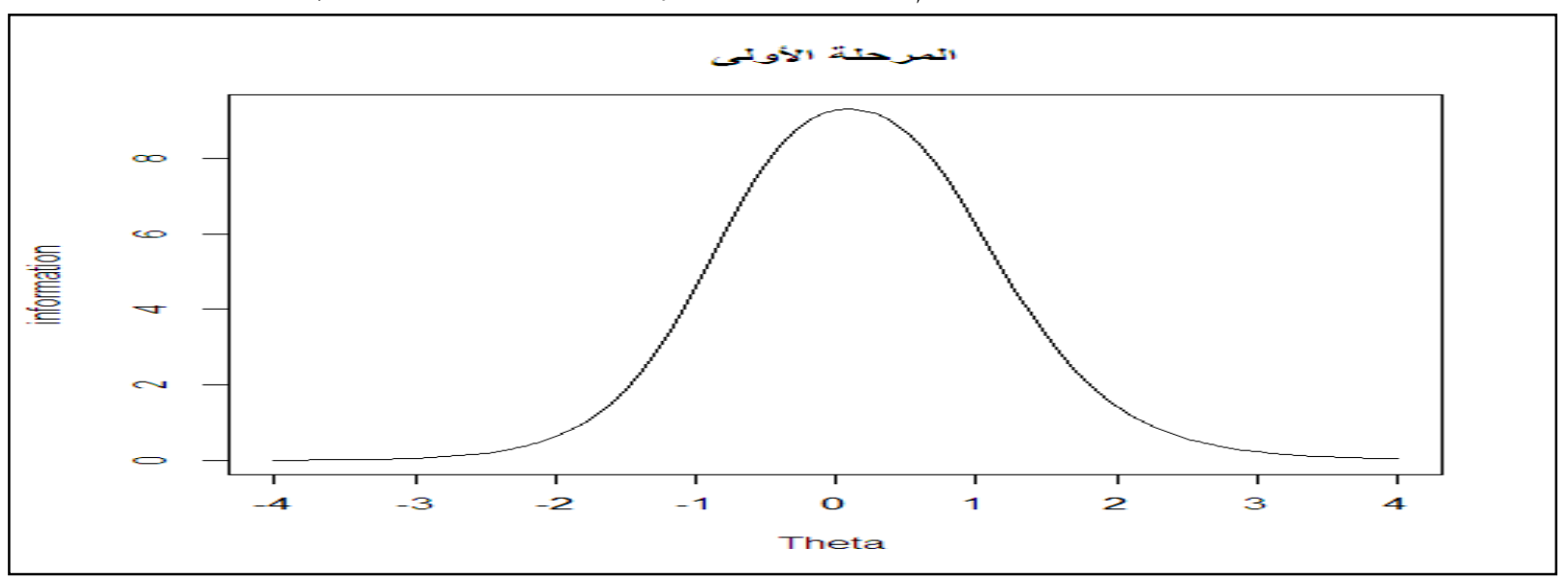



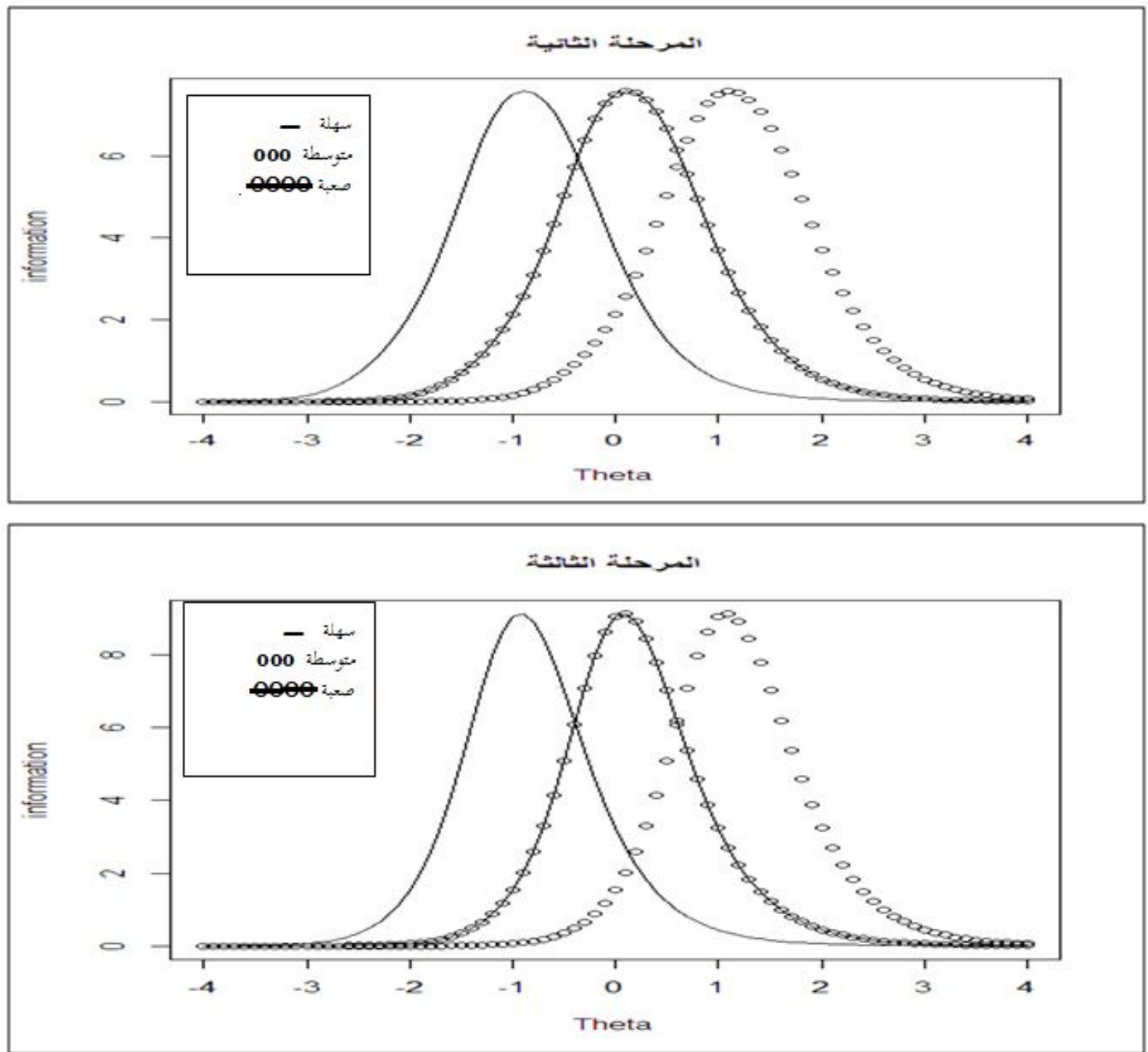

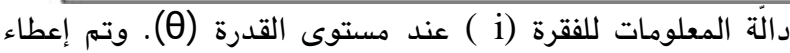
طول الاختبار الرمز (N) . ويعرف متغير القرار كالآتي:

$$
X_{\text {if }}=\left\{\begin{array}{c}
f \text { إذا كانت الفقرة متضمنة في النموذج (3) } 0 \\
\text { : خلاف ذلك } 0
\end{array}\right.
$$

$\operatorname{Min} Z$

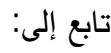

$$
\text { لكل قيم }
$$

$\sum_{i=1}^{\mathrm{I}} \mathrm{I}_{\mathrm{i}}(\theta) \mathrm{X}_{\mathrm{if}} \leq \mathrm{T}_{\theta}+\mathrm{Z}$ ولجميع النماذج)

$$
\text { لكل قيم }
$$

$\sum_{i=1}^{\mathrm{I}} \mathrm{I}_{\mathrm{i}}(\theta) \mathrm{X}_{\mathrm{if}} \geq \mathrm{T}_{\theta}+\mathrm{Z}$ ولجميع النماذج) $\sum_{f=1}^{5} X_{i f} \leq 1$ لكل قيم

$\sum_{i \in V_{c}} X_{i f} \geq n_{c}$ ( ولجميع النمان
في الثكل (3)، كان طول الوحدة في المرحلة الأولى (وحدة

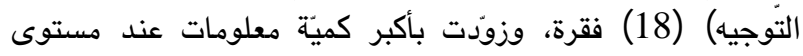

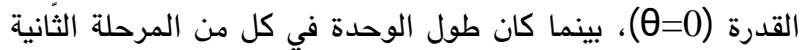

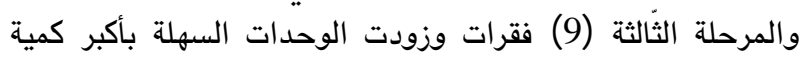

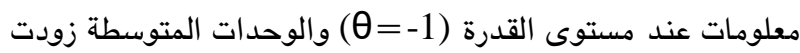
بأكبر كمية معلومات عند مستوى قدرة (

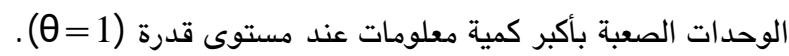

وتمز استخدام البرمجة مختلطة العدد الصحيح (Mixed-) Diao \& van der ) (Integer Programming: MIP (Linden, 2011

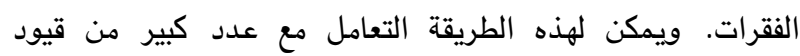

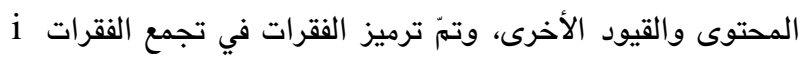
تاني

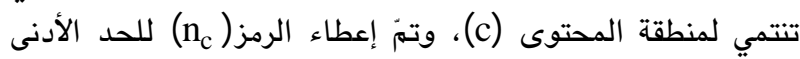

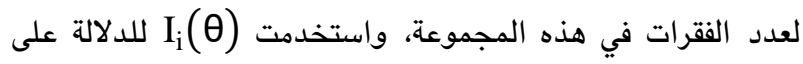




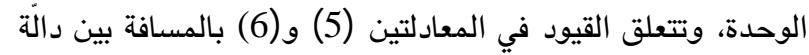

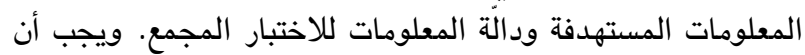

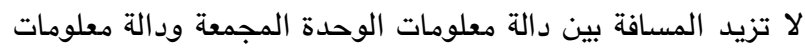

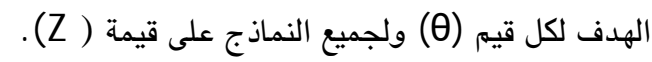

تمَ بناء الوحدات باستخدام استراتيجية من (أسفل إلى أعلى)

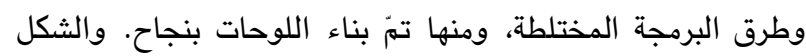
(4) يعرض دوال المعلومات لوحدة التوجيه لخمسة ألواح.

$$
\begin{aligned}
& \sum_{i=1}^{I} X_{i f}=N \text { لكل النماذج) } \\
& X_{\mathrm{i}} \in\{0,1\} \\
& Z \geq 0
\end{aligned}
$$

ويتعلق القيد في المعادلة (7) بعدم تداخل الفقرات بين

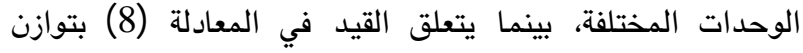

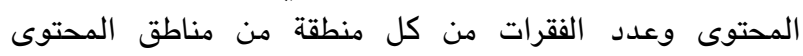
المتضمنة في كل وحدة، ويتعلق القيد في المعادلة (9) بطول من لمن

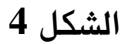

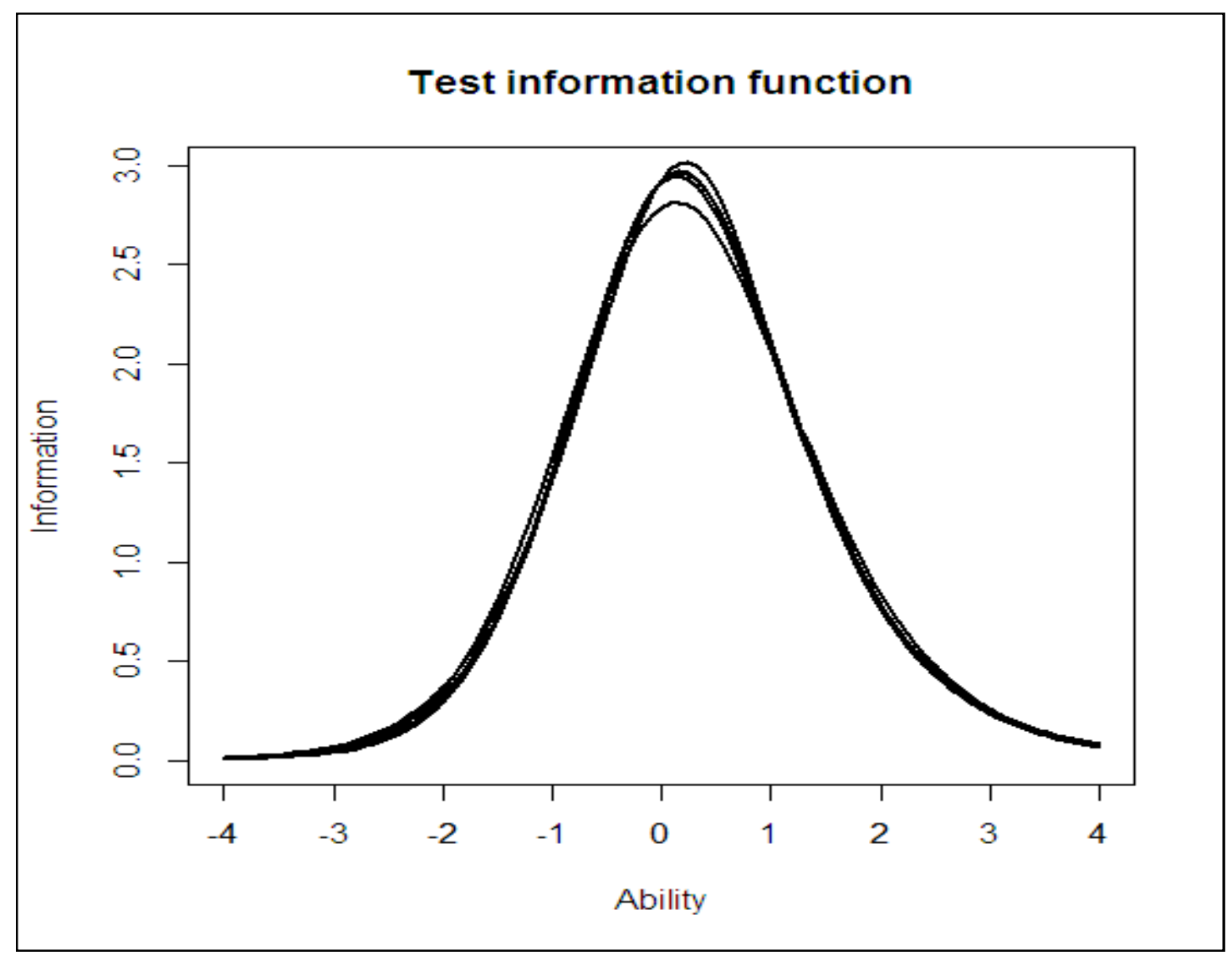

للمفحوص والوحدة التي ستقدم له في المرحلة التالية ( Luecht .(et al., 2006

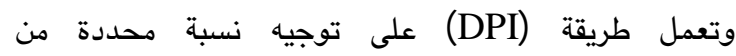

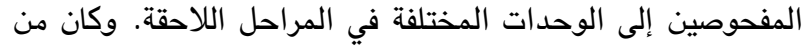

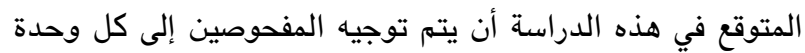

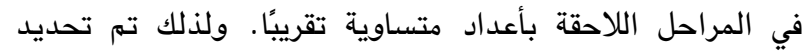

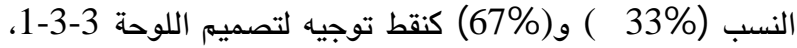

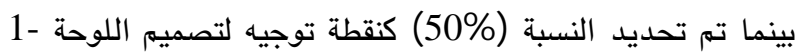

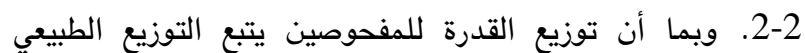

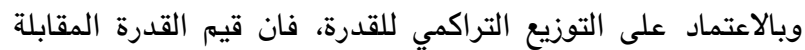
للنسب السابقة تساوي (0.43 -) و (0.43) و و(0) على التوالي.
يوضح الثكل (4) دوال المعلومات لوحدات التوجيه لجميع

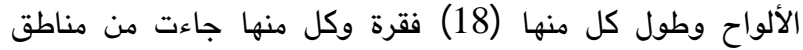

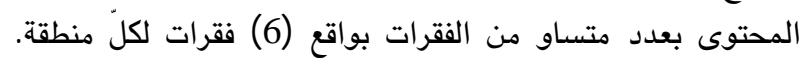

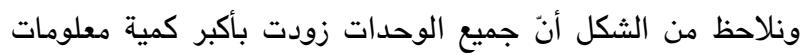

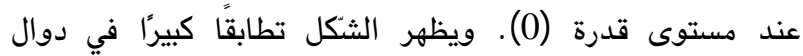

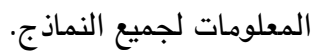
واستخدمت الدراسة الحالية طريقة المعلومات القصوى (DPI)

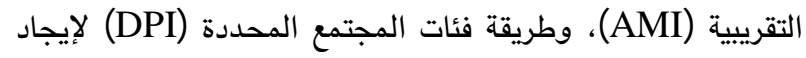

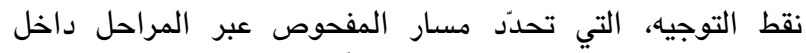
اللوحة، وطريقة (AMI) تستخدم دالة معلومات الاختبار لتحديد نقط التوجيه من خلال تحديد نقطة التقاطع بين الوحدة التي تقدم 
النظر إلى الفروق في قيم (MSE)، وقيم متوسنط التحيَز التي تقل

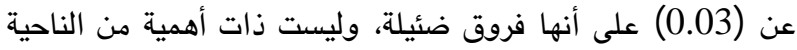

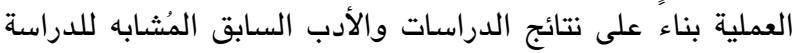

الحالية (Chang \& Ying, 1999; Wang, 2017).

$$
\text { نتائج الدراسة }
$$

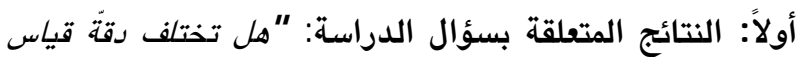

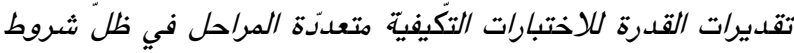

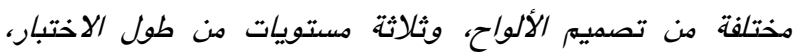

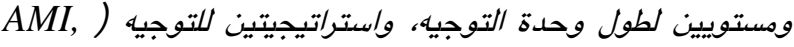
$\varphi^{\prime \prime}(D P I$

للإجابة عن سؤال الدراسة؛ تمّ تقييم دقة القياس من خلال

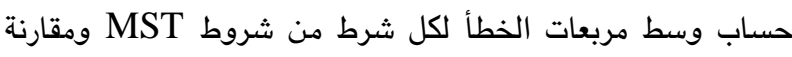
النتائج، كما يظهر في الجداول (3،4، ) .

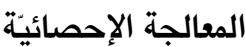

تمز الحكم على دقة قياس تصاميم MST المختلفة من خلال

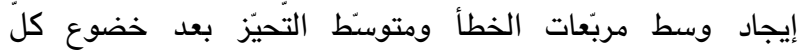

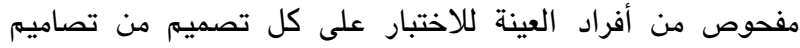
MST وتكرار الاختبار (10) مرات على جميع أفراد العينة، ومعادلة

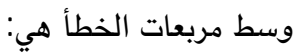

$$
M S E=\sum_{\mathrm{i}=1}^{\mathrm{N}} \frac{\left(\theta_{\mathrm{i}}-\hat{\theta}_{\mathrm{i}}\right)^{2}}{\mathrm{~N}}
$$

ومعادلة متوسنط التحيَز هي: - مي

$$
\text { Mean Bias }=\sum_{\mathrm{i}=1}^{\mathrm{N}} \frac{\left(\theta_{i}-\widehat{\theta}_{i}\right)}{\mathrm{N}}
$$

حيث N عدد المفحوصين، للمفحوصين، القياس كلما اقتربت (MSE) ومتوسط التحيز من الصفر، ويمكن

\begin{tabular}{|c|c|c|c|c|}
\hline DPI & AMI & التصميم & طول وحدة التوجيه & طول الاختبار \\
\hline 0.1114 & 0.1123 & $1-3-3$ & 18 & 36 \\
\hline 0.1107 & 0.1109 & $1-2-2$ & 18 & 36 \\
\hline 0.1095 & 0.1125 & $1-3-3$ & 9 & 36 \\
\hline 0.1114 & 0.1133 & $1-2-2$ & 9 & 36 \\
\hline
\end{tabular}
الجدول

وسط مريّات الخطأ لتقدير القدرة لاختبار بطول 36 فقرة.

2-2 ) باختلاف طريقتي التوجيه (DPI,AMI) وطول وحدة (0,9)

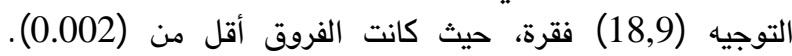

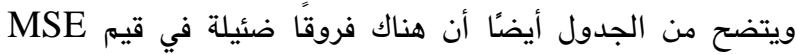

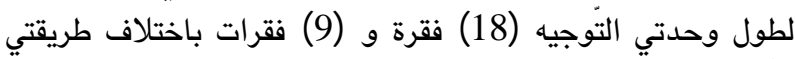

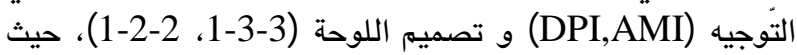
كانت الفروق أقل من (0.002) .
يظهر الجدول (3) وسط مربعات الخطأ لجميع شروط MST

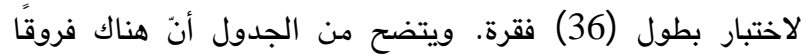

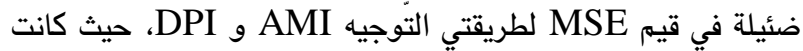

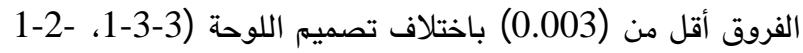

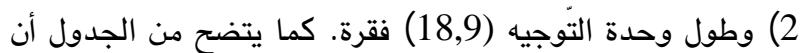

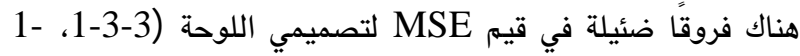

\begin{tabular}{|c|c|c|c|c|}
\hline DPI & AMI & التصميم & طول وحدة التوجيه & طول الاختبار \\
\hline 0.1432 & 0.1435 & $1-3-3$ & 12 & 24 \\
\hline 0.1413 & 0.1415 & $1-2-2$ & 12 & 24 \\
\hline 0.1434 & 0.1416 & $1-3-3$ & 6 & 24 \\
\hline 0.1421 & 0.1382 & $1-2-2$ & 6 & 24 \\
\hline
\end{tabular}

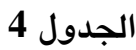

وسط مربعات الخطأ لتقدير القدرة لاختبار بطول 24 فقرة.

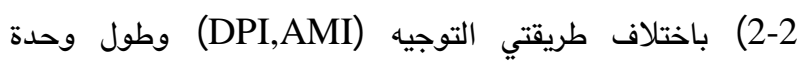
التوجيه (12,6) فقرة، حيث كانت الفروق أقل من (D) (0.0034).

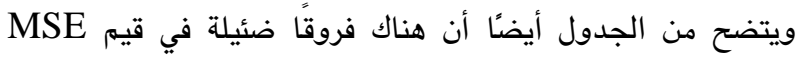

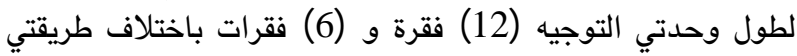

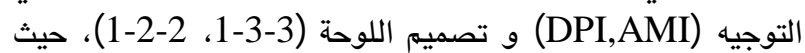

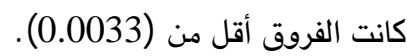

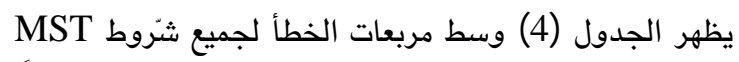
لاختبار بطول (24) فقرة، ويتضح من الجدول أن هناك فناك فروطاً

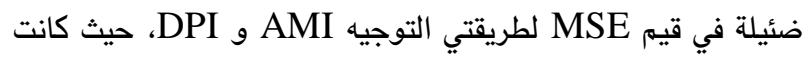

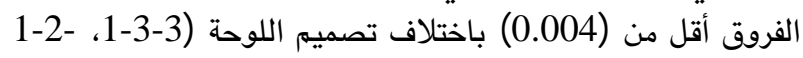

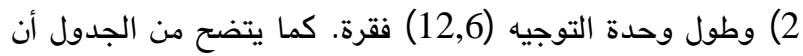

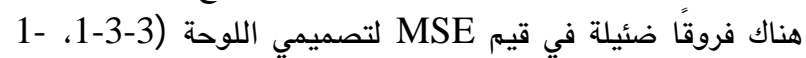


وسط مريّعات الخطأ لتقدير القدرة لاختبار بطول 12 فقرة.

\begin{tabular}{|c|c|c|c|c|}
\hline DPI & AMI & التصميم & طول وحدة التوجيه & طول الاختبار \\
\hline 0.2322 & 0.2478 & $1-3-3$ & 6 & 12 \\
\hline 0.2251 & 0.2245 & $1-2-2$ & 6 & 12 \\
\hline 0.2220 & 0.2220 & $1-3-3$ & 3 & 12 \\
\hline 0.2231 & 0.2224 & $1-2-2$ & 3 & 12 \\
\hline
\end{tabular}

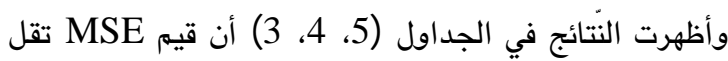

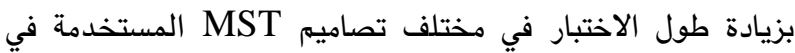

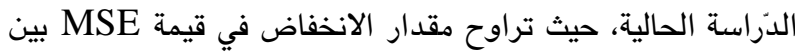

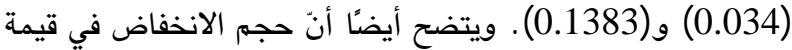
MSE

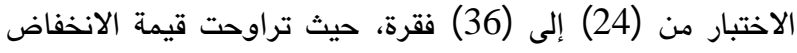

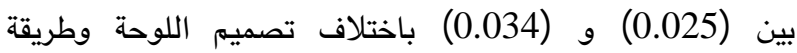

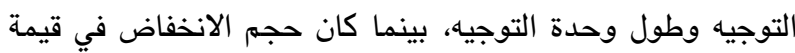
MSE الاختبار من (12) فقرة إلى (24) فقرة.

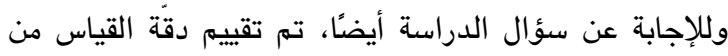

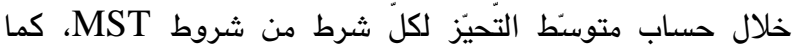
يظهر في الجداول (6، 7، 8. 8) .

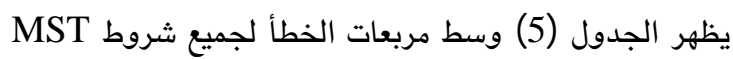

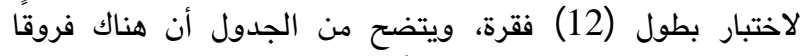

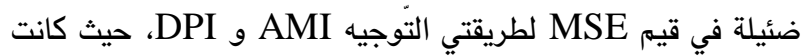

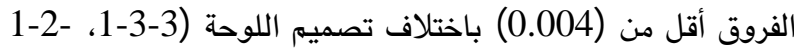

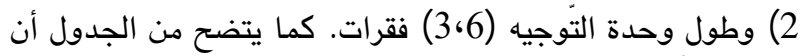

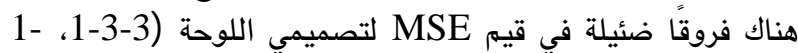

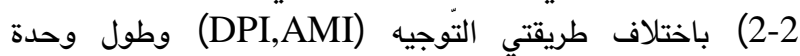

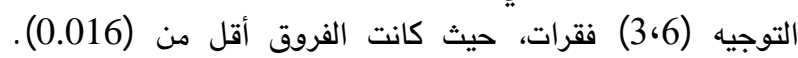

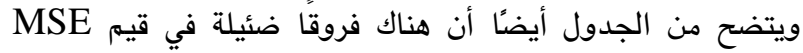

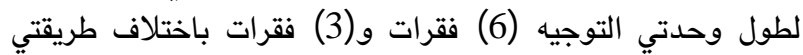

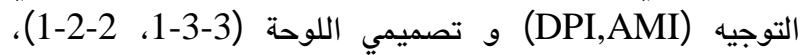
حيث كانت الفروق أقل من (0.026).

الجدول 6

متوسّط التحيزّ لتقدير القدرة لاختبار بطول 36 نقرة.

\begin{tabular}{ccccc}
\hline DPI & AMI & طول الاختبار & 36 \\
\hline-0.001 & -0.001 & $1-3-3$ & 18 & 36 \\
0.001 & -0.001 & $1-2-2$ & 18 & 36 \\
-0.003 & -0.002 & $1-3-3$ & 9 & 36 \\
-0.001 & -0.001 & $1-2-2$ & 9 & 36 \\
\hline
\end{tabular}

التحيز لاستراتيجية التوجيه بين (0.001) و (0.002)، وتراوحت

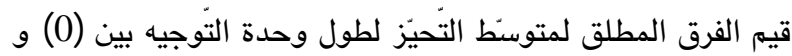
(0.001)

يظهر الجدول (6) أن قيم متوسنط التحيّز متثابهة بشكل كبير ولا يوجد فرق واضح بين تصميم اللوحة وطريقة التوجيه وطول وحدة التوجيه لاختبار بطول (36) فقرة وجميعها قريبة من الصفر، لونه

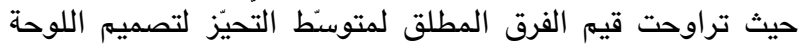
بين (0.001) و (0.002)، وتراوحت قيم الفرق المطلق لمتوسط لتمبط

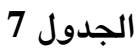

متوسيّ التحيزّ لتقدير القدرة لاختبار بطول 24 نقرة.

\begin{tabular}{ccccc}
\hline DPI & AMI & طول 24 \\
\hline 0.001 & 0.004 & $1-3-3$ & 12 & 24 \\
-0.001 & 0.001 & $1-2-2$ & 12 & 24 \\
-0.001 & 0.001 & $1-3-3$ & 6 & 24 \\
0.002 & -0.001 & $1-2-2$ & 6 & 24 \\
\hline
\end{tabular}


المطلق لمتوسنط التحيَز لاستراتيجية التوجيه بين (0.001) و(0.003)، وتراوحت قيم الفرق المطلق لمتوسنّ التِّيّز لطول

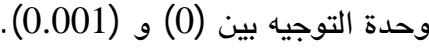

يتضح من الجدول (7) أنّ قيم متوسنط التحيّز متشابهة بشكل كبير ولا يوجد فرق واضح بين تصميم اللوحة وطريقة

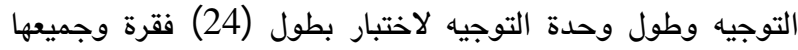
قريبة من الصفر، حيث تراوحت قيم الفرق المطلق لمتوسيط التحيّز

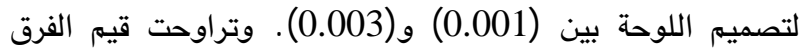

الجدول 8

متوسنّ التَحيزّ لتقدير القدرة لاختبار بطول 12 فقرة.

\begin{tabular}{|c|c|c|c|c|}
\hline DPI & AMI & التصميم & طول وحدة التوجيه & طول الاختبار \\
\hline 0.005 & -0.002 & $1-3-3$ & 6 & 12 \\
\hline-0.002 & 0.001 & $1-2-2$ & 6 & 12 \\
\hline-0.003 & -0.002 & $1-3-3$ & 3 & 12 \\
\hline-0.001 & -0.009 & $1-2-2$ & 3 & 12 \\
\hline
\end{tabular}

آخر، فقد كانت صعوبة الفقرات للوحدات في المرحلتين الثَانية

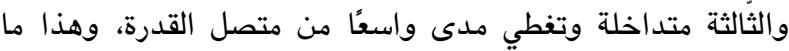
أكدت عليه نتائج دراسة كيم وآخرين (Kim et al., 2013). وأظهرت النتائج أيضًا أنّ قيمة MSE ومتوسنط التحيّز كانت

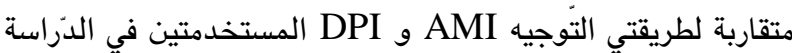

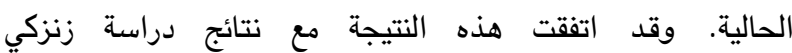
(Kim et al., 2013) ودراسة كيم وآخرين (Zenisky, 2004) Alghamdi, ) ودراسة وانغ (Wang, 2017) ودراسة الغامدين (Zenisk (2018

وأظهرت النتائج أن قيمة MSE ومتوسنط التَّيَز كانت متقاربة باختلاف طول وحدة التوجيه مع ثبات طول الاختبار . وقد تثابهت

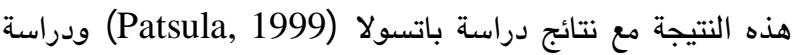
تشنغ وآخرين (Zheng et al., 2012) ودراسة كيم وآخرين

.(Kim et al., 2015)

وتعارضت نتائج الدزاسة الحالية مع نتائج دراسة اوزتورك

(Öztürk, 2019)

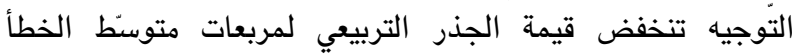

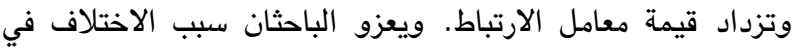

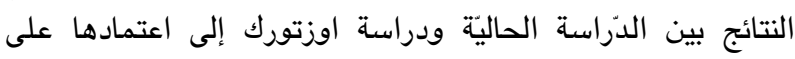

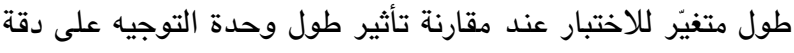

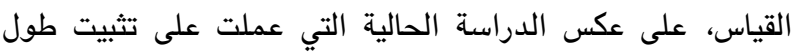

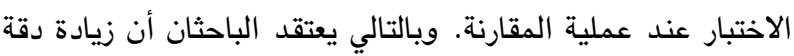

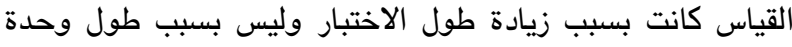

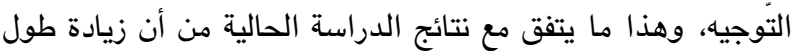
وحدة التوجية تؤدي لزيادة في طول الاختبار، وكون الفقرات يتم

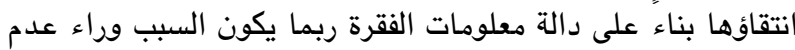
وجود فروق واضحة في قيمة MSE ومتوسنط التحيَّ .
يظهر الجدول (8) أنَ قيم متوسنط التحيَّ متشابهة ولا يوجد فرق واضح بين تصميم اللوحة وطريقة التوجيه وطول وحدة التهدة

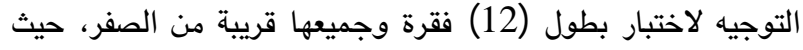

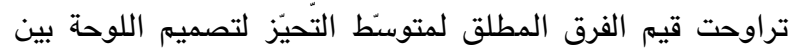
(0.001) و(0.007)، وتراوحت قيم الفرق المطلق لمتوسئط التحيَّ

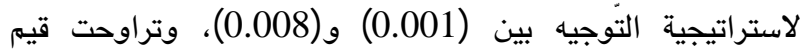

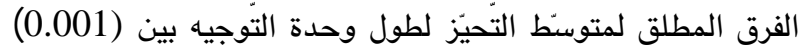

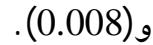

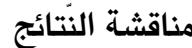

بالاعتماد على نتائج سؤال الدراسة التي أظهرت أن قيم MSE

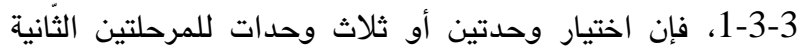

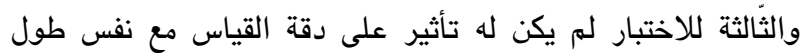

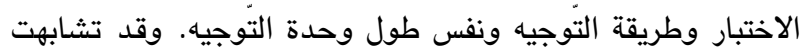

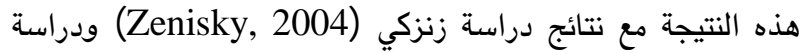
زنزكي وهامبلتون (Zenisky \& Hambleton, 2004) ودنج (Zim) ودراسة كيم وآخرين (Kim et al., 2013) ودراسة وانغ (

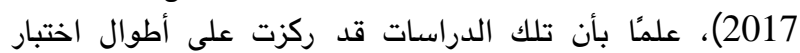
تتراوح بين المتوسطة والكبيرة، في حين استخدمت الدراسة الحالية

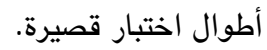

ويعزو الباحثان هذه النتيجة إلى طريقة تحديد نقطة المركز لدالة معلومات الهدف للوحدات في المرحلتين الثانية والثالثة لكلا

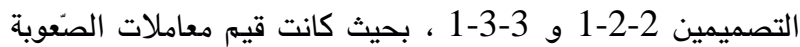

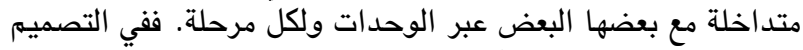

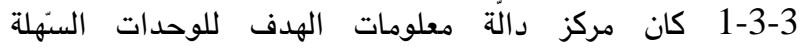

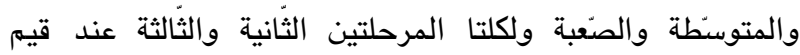

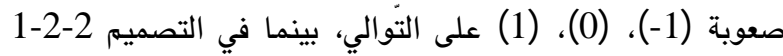
كانت نقط المركز للوحدات السهلة والصعبة ولكلتا المرحلتين الثانية التئ

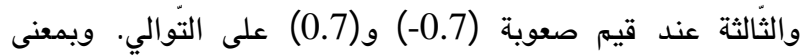


(10) مرات على جميع أفراد العينة، إلى أداء أفضل للاختبار

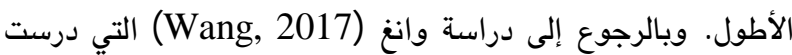

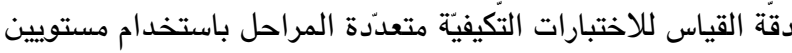

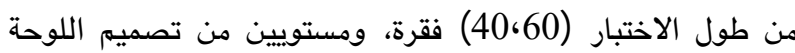

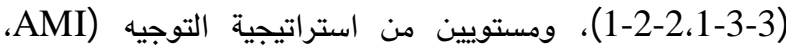

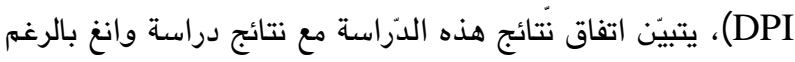

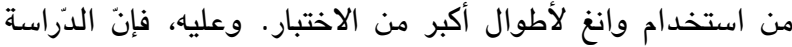

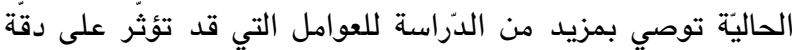

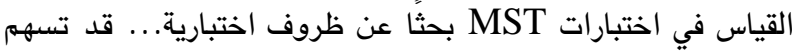

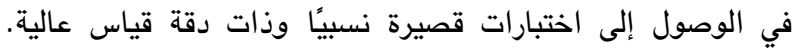

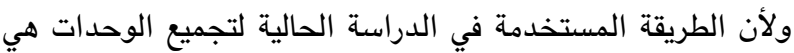

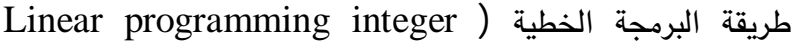

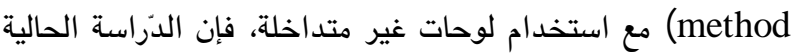

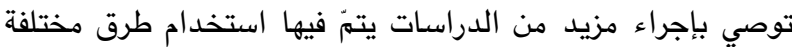
لتجميع الوحدات، وكذلك استخدام تصميم لوحات متداخلة. وفي ضوء النتائج التي انتهت إليها هذه الدراسة، يوصي

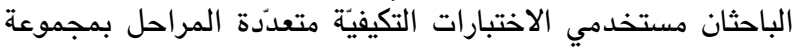

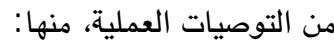
• استخدام طريقة التوجيه (DPI) بدلاً من طريقة التوجيه (AMI) ، لسهولة تحديد نقط التوجيه وفق هذه الطريقة مقارنة

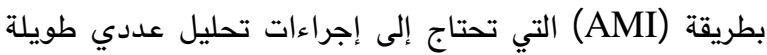
ومعقدة.

• استخدام تصميم اللوحة 2-2-1-3، مع وحدة توجيه أطول، بدلاً

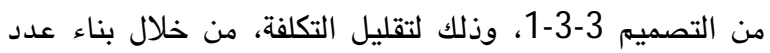

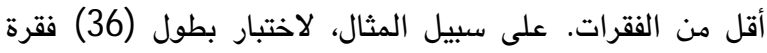

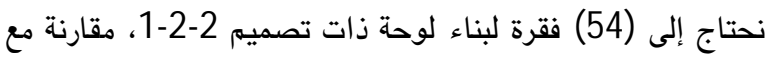

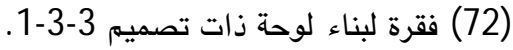

وأظهرت نتائج الدراسة أن قيمة MSE ومتوسنط التحيّز تقل

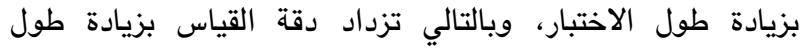

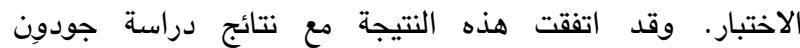
Jodoin et al., ) ودراسة جودون وآخرين (Jodoin, 2003)

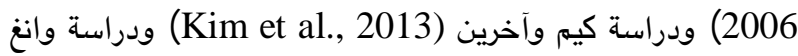
Sari \& ) ودراسة ساري وهوغنز مانلي (Wang, 2017) .(Huggins-Manley, 2017

ويعزو الباحثان هذه التيجة إلى الصيغة الرياضيّة لدالة

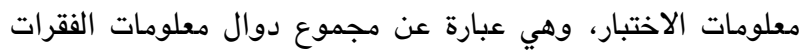

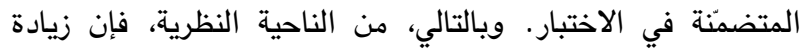
طول الاختبار تؤدي إلى زيادة كمية المعلومات على طولى طول متصنّل

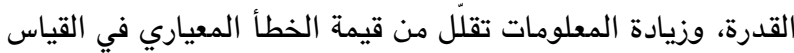
وتعمل على زيادة دقة تقدير القدرة.

ومن ناحية عملية، فإنَ زيادة طول الاختبار بشكل كبير تؤديّي

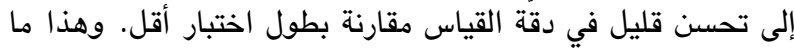

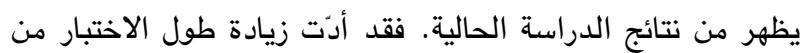

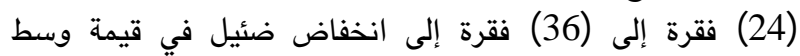

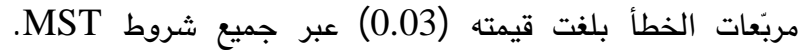

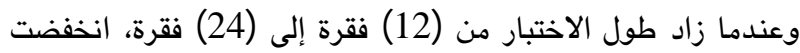
قيمة وسط مربُعات الخطأ بشكل أكبر، حيث تراوحت قيم الآن الانخفاض

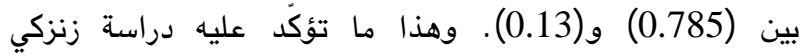

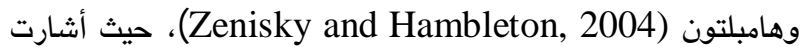

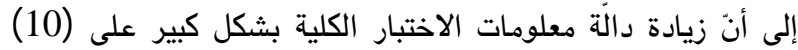

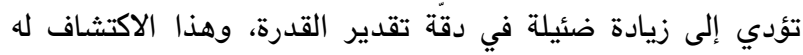

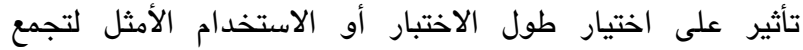

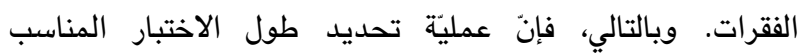

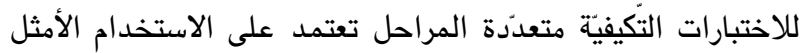

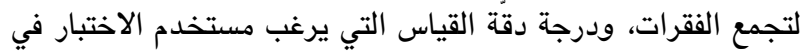

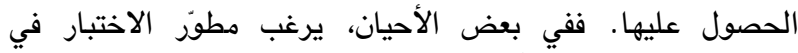
اختبارات قصيرة مع دقة قياس مقبولة، وفي بعض الأحيان تكون درجة دقة القياس هي محطُ اهتمام مطورّ الاختبار . الاستنتاجات والتّوصيات

بينت النتائج أنّ عدد فقرات الاختبار هي العامل الأكثر تأثيرًا

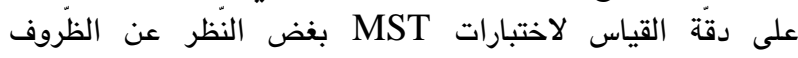

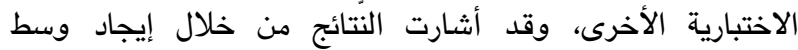

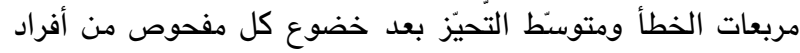
العينة للاختبار على كل تصميم من تصاميم MST وتكريم التهرار الاختبار 


\section{References}

Alghamdi, H. (2018). Assessment of multiple-form structure designs of multistage testing using IRT. Ph.D. Dissertation, University of Denver, U.S.A.

Armstrong, R., \& Roussos, L. (2003). A method to determine targets for multi-stage adaptive tests. (Computerized Testing Rep. No. 02-07). Law School Admission Council.

Chang, H., \& Ying, Z. (1999). A-stratified multistage computerized adaptive testing. Applied Psychological Measurement, 23(3), 211-222.

Diao, Q. \& van der Linden, W. (2011). Automated test assembly using lp_solve version 5.5 in R. Applied Psychological Measurement, 35(5), 398-409.

Jodoin, M. (2003). Measurement efficiency of innovative item formats in computer-based testing. Journal of Educational Measurement, 40(1), 1-15.

Jodoin, M., Zenisky, A., \& Hambleton, R. (2006). Comparison of the psychometric properties of several computer-based test designs for credentialing exams with multiple purposes. Applied Measurement in Education, 19(3), 203-220.

Kim, J., Chung, H., Park, R., \& Dodd, B. (2013). A comparison of panel designs with routing methods in the multistage test with the partial credit model. Behavior Research Methods, 45(4), 1087-1098.

Kim, S., Moses, T., \& Yoo, H. (2015). A comparison of IRT proficiency estimation methods under adaptive multistage testing. Journal of Educational Measurement, 52(1), 70-79.

Leung, C., Chang, H., \& Hau, K. (2005). Computerized adaptive testing: A mixture item selection approach for constrained situations. British Journal of Mathematical and Statistical Psychology, 58(2), 239-257.

Lord, F. M. (1980). Application of item response theory to practical testing problems. Lawrence Erlbaum Associates, Inc.
Luecht, R. (2000, April). Implementing the computer-adaptive sequential testing (CAST) framework to mass produce high-quality computer-adaptive and mastery tests. In: Annual Meeting of National Council on Measurement in Education. New Orleans, LA.

Luecht, R., \& Nungester, R. (1998). Some practical examples of computer-adaptive sequential testing. Journal of Educational Measurement, 35, 229-249. Retrieved from: https://doi-

org.du.idm.oclc.org/10.1111/j.1745-3984. 1998.tb00537.x

Luecht, R., Brumfield, T., \& Breithaupt, K. (2006). A testlet assembly design for adaptive multistage tests. Applied Measurement in Education, 19(3), 189-202.

Magis, D., Yan, D., \& Von Davier, A. (2017). Computerized adaptive and multistage testing with $R$ : Using packages catR and mstR. Springer.

Melican, G., Breithaupt, K., \& Zhang, Y. (2009). Designing and implementing a multistage adaptive test: The uniform CPA exam. In: W. J. van der Linden \& C.W. Glas (Eds.). Elements of adaptive testing (pp. 167-190). Springer.

National Center. for Assessment in Higher Education. (2017). General aptitude test (GAT)

Öztürk, N. (2019). How the length and characteristics of routing module affect ability estimation in ca-MST. Universal Journal of Educational Research, 7(1), 164-170.

Patsula, L. (1999). A comparison of computerized adaptive testing and multi-stage testing. Ph.D. Dissertation, University of Massachusetts, Amherst.

Rudner, L. (2009). Implementing the graduate management admission test computerized adaptive test. In: J. van der Linden \& A. W. Glas. (Eds). Elements of adaptive testing (pp. 151-165). Springer. 
Sari, H., \& Huggins-Manley, A. (2017). Examining content control in adaptive tests: Computerized adaptive testing vs. computerized adaptive multistage testing. Educational Sciences: Theory \& Practice, 17(5), 1759-1781.

Swanson, L., \& Stocking, M. (1993). A model and heuristic for solving very large item selection problems. Applied Psychological Measurement, 17(2), 151-166.

van der Linden, W., Ariel, A., \& Veldkamp, B. (2006). Assembling a computerized adaptive testing item pool as a set of linear tests. Journal of Educational and Behavioral Statistics, 31(1), 81-99.

Wainer, H. (2000). Computerized adaptive testing: A primer. Lawrence Erlbaum Associates, Inc.

Wang, K. (2017). A fair comparison of the performance of computerized adaptive testing and multistage adaptive testing. (Ph.D. Dissertation, University of Michigan State, U.S.A).

Wang, X., Fluegge, L., \& Luecht, R. (2012). A large-scale comparative study of the accuracy and efficiency of ca-MST panel design configurations. In: Annual Meeting of the National Council on Measurement in Education. April 2012, Vancouver, BC, Canada.
Yan, D., Lewis, C. \& Von Davier, A. (2014).Overview of computerized multistage test. In: D. Yan, A. von Davier \& C. Lewis (Eds.). Computerized multistage testing: Theory and applications (pp.3-20). Chapman and Hall/CRC.

Zenisky, A. (2004). Evaluating the effects of several multi-stage testing design variables on selected psychometric outcomes for certification and licensure assessment. (Ph.D. Dissertation, University of Massachusetts Amherst, U.S).

Zenisky, A., \& Hambleton, R. (2004). Effects of selected multi-stage test design alternatives on credentialing examination outcomes. Paper presented at the Annual Meeting of the National Council on Measurement in Education, April 2004, San Diego, CA.

Zheng, Y., \& Chang, H. (2015). On-the-fly assembled multistage adaptive testing. Applied Psychological Measurement, 39(2), 104-118.

Zheng, Y., Nozawa, Y., Gao, X., \& Chang, H. (2012). Multistage adaptive testing for a large-scale classification test: Design, heuristic assembly and comparison with other testing modes (ACT Research Report 6). Retrieved from: https//pdfs. Semanticscholar. org/049b/54fca400fb73116c438a1f834adcc34 9f07e.pdf. 